\title{
ANALYSIS OF THE MORLEY ELEMENT FOR THE CAHN-HILLIARD EQUATION AND THE HELE-SHAW FLOW *
}

\author{
SHUONAN WU ${ }^{\dagger}$ AND YUKUN LI ${ }^{\ddagger}$
}

\begin{abstract}
The paper analyzes the Morley element method for the Cahn-Hilliard equation. The objective is to derive the optimal error estimates and to prove the zero-level sets of the CahnHilliard equation approximate the Hele-Shaw flow. If the piecewise $L^{\infty}\left(H^{2}\right)$ error bound is derived by choosing test function directly, we cannot obtain the optimal error order, and we cannot establish the error bound which depends on $\frac{1}{\epsilon}$ polynomially either. To overcome this difficulty, this paper proves them by the following steps, and the result in each next step cannot be established without using the result in its previous one. First, it proves some a priori estimates of the exact solution $u$, and these regularity results are minimal to get the main results; Second, it establishes $L^{\infty}\left(L^{2}\right)$ and piecewise $L^{2}\left(H^{2}\right)$ error bounds which depend on $\frac{1}{\epsilon}$ polynomially based on the piecewise $L^{\infty}\left(H^{-1}\right)$ and $L^{2}\left(H^{1}\right)$ error bounds; Third, it establishes piecewise $L^{\infty}\left(H^{2}\right)$ optimal error bound which depends on $\frac{1}{\epsilon}$ polynomially based on the piecewise $L^{\infty}\left(L^{2}\right)$ and $L^{2}\left(H^{2}\right)$ error bounds; Finally, it proves the $L^{\infty}\left(L^{\infty}\right)$ error bound and the approximation to the Hele-Shaw flow based on the piecewise $L^{\infty}\left(H^{2}\right)$ error bound. The nonstandard techniques are used in these steps such as the generalized coercivity result, integration by part in space, summation by part in time, and special properties of the Morley elements. If one of these techniques is lacked, either we can only obtain the sub-optimal piecewise $L^{\infty}\left(H^{2}\right)$ error order, or we can merely obtain the error bounds which are exponentially dependent on $\frac{1}{\epsilon}$. The approach used in this paper provides a way to bound the errors in higher norm from the errors in lower norm step by step, which has a profound meaning in methodology. Numerical results are presented to validate the optimal $L^{\infty}\left(H^{2}\right)$ error order and the asymptotic behavior of the solutions of the Cahn-Hilliard equation.
\end{abstract}

Key words. Morley element, Cahn-Hilliard equation, generalized coercivity result, $\frac{1}{\epsilon}$ polynomial dependence, Hele-Shaw flow

AMS subject classifications. 65N12, 65N15, 65N30

1. Introduction. Consider the following Cahn-Hilliard equation with Neumann boundary conditions:

$$
\begin{aligned}
u_{t}+\Delta\left(\epsilon \Delta u-\frac{1}{\epsilon} f(u)\right) & =0 & & \text { in } \Omega_{T}:=\Omega \times(0, T], \\
\frac{\partial u}{\partial n}=\frac{\partial}{\partial n}\left(\epsilon \Delta u-\frac{1}{\epsilon} f(u)\right) & =0 & & \text { on } \partial \Omega_{T}:=\partial \Omega \times(0, T], \\
u & =u_{0} & & \text { in } \Omega \times\{t=0\},
\end{aligned}
$$

where $\Omega \subseteq \mathbf{R}^{2}$ is a bounded domain, $f(u)=u^{3}-u$ is the derivative of a double well potential $F(u)$ which is defined by

$$
F(u)=\frac{1}{4}\left(u^{2}-1\right)^{2} .
$$

The Allen-Cahn equation [3, 6, 12, 20, 17, 16, 19, 24] and the Cahn-Hilliard equation $[2,12,25,29]$ are two basic phase field models to describe the phase transition process. They are also proved to be related to geometric flow. For example, the zero-level sets of the Allen-Cahn equation approximate the mean curvature [15, 24] and the zero-level sets of the Cahn-Hilliard equation approximate the Hele-Shaw flow [28, 2]. The CahnHilliard equation was introduced by J. Cahn and J. Hilliard in [11] to describe the

* The work of Shuonan Wu is partially supported by the startup grant from Peking Unversity.

${ }^{\dagger}$ School of Mathematical Sciences, Peking University, China, 100871 (snwu@math.pku.edu.cn)

${ }^{\ddagger}$ Department of Mathematics, The Ohio State University, Columbus, U.S.A. (1i.7907@osu.edu) 
process of phase separation, by which the two components of a binary fluid separate and form domains pure in each component. It can be interpreted as the $H^{-1}$ gradient flow [2] of the Cahn-Hilliard energy functional

$$
J_{\epsilon}(v):=\int_{\Omega}\left(\frac{\epsilon}{2}|\nabla v|^{2}+\frac{1}{\epsilon} F(v)\right) \mathrm{d} x .
$$

There are a few papers $[4,30,13,14]$ discussing the error bounds, which depend on the exponential power of $\frac{1}{\epsilon}$, of the numerical methods for Cahn-Hilliard equation. Such an estimate is clearly not useful for small $\epsilon$, in particular, in addressing the issue whether the computed numerical interfaces converge to the original sharp interface of the HeleShaw problem. Instead, the polynomial dependence in $\frac{1}{\epsilon}$ is proved in [21, 22] using the standard finite element method, and in [18, 26] using the discontinuous Galerkin method. Due to the high efficiency of the Morley elements, compared with mixed finite element methods or $C^{1}$-conforming finite element methods, the Morley finite element method is used to derive the error bound which depends on $\frac{1}{\epsilon}$ polynomially in this paper.

The highlights of this paper are fourfold. First, it establishes the piecewise $L^{\infty}\left(L^{2}\right)$ and $L^{2}\left(H^{2}\right)$ error bounds which depend on $\frac{1}{\epsilon}$ polynomially. If the standard technique is used, we can only prove that the error bounds depend on $\frac{1}{\epsilon}$ exponentially, which can not be used to prove our main theorem. To prove these bounds, special properties of the Morley elements are explored, i.e., Lemma 2.3 in [14], and piecewise $L^{\infty}\left(H^{-1}\right)$ and $L^{2}\left(H^{1}\right)$ error bounds [27] are required. Second, by making use of the piecewise $L^{\infty}\left(L^{2}\right)$ and $L^{2}\left(H^{2}\right)$ error bounds above, it establishes the piecewise $L^{\infty}\left(H^{2}\right)$ error bound which depends on $\frac{1}{\epsilon}$ polynomially. If the standard technique is used, we can only get the error bound in Remark 2, which does not have an optimal order. The crux here is to employ the summation by part in time and integration by part in space techniques simultaneously to handle the nonlinear term, together with the special properties of the Morley elements. Third, the minimal regularity of $u$ is used, i.e., $\left\|u_{t t}\right\|_{L^{2}\left(L^{2}\right)}$ regularity instead of $\left\|u_{t t}\right\|_{L^{\infty}\left(L^{2}\right)}$ regularity is used, and the a priori estimate is derived in Theorem 2.2. Fourth, the $L^{\infty}\left(L^{\infty}\right)$ error bound is established using the optimal piecewise $L^{\infty}\left(H^{2}\right)$ error, by which the main result that the zero-level sets of the Cahn-Hilliard equation approximate the Hele-Shaw flow is proved in Section 5.

The organization of this paper is as follows. In Section 2, the standard Sobolev space notation is introduced, some useful lemmas are stated, and a new a priori estimate of the exact solution $u$ is derived. In Section 3, the fully discrete approximation based on the Morley finite element space is presented. In Section 4, first the polynomially dependent piecewise $L^{\infty}\left(L^{2}\right)$ and $L^{2}\left(H^{2}\right)$ error bounds are established based on piecewise $L^{\infty}\left(H^{-1}\right)$ and $L^{2}\left(H^{1}\right)$ error bounds, then the polynomially dependent piecewise $L^{\infty}\left(H^{2}\right)$ error bound is established based on piecewise $L^{\infty}\left(L^{2}\right)$ and $L^{2}\left(H^{2}\right)$ error bounds, by which the $L^{\infty}\left(L^{\infty}\right)$ error bound is proved. In Section 5 , the approximation of the zero-level sets of the Cahn-Hilliard equation of the Hele-Shaw flow is proved. In Section 6, numerical tests are presented to validate our theoretical results, including the optimal error orders and the approximation of the Hele-Shaw flow.

2. Preliminaries. In this section, we present some results which will be used in the following sections. Throughout this paper, $C$ denotes a generic positive constant which is independent of interfacial length $\epsilon$, spacial size $h$, and time step size $k$, and it may have different values in different formulas. The standard Sobolev space notation below is used in this paper. 


$$
\begin{aligned}
& \|v\|_{0, p, A}=\left(\int_{A}|v|^{p} \mathrm{~d} x\right)^{1 / p} \quad 1 \leq p<\infty, \\
& \|v\|_{0, \infty, A}=\operatorname{ess} \sup _{A}|v|, \\
& |v|_{m, p, A}=\left(\sum_{|\alpha|=m}\left\|D^{\alpha} v\right\|_{0, p, A}^{p}\right)^{1 / p} \quad 1 \leq p<\infty, \\
& \|v\|_{m, p, A}=\left(\sum_{j=0}^{m}|v|_{m, p, A}^{p}\right)^{1 / p}
\end{aligned}
$$

Here $A$ denotes some domain, i.e., a single mesh element $K$ or the whole domain $\Omega$. When $A=\Omega,\|\cdot\|_{H^{k}},\|\cdot\|_{L^{k}}$ are used to denote $\|\cdot\|_{H^{k}(\Omega)},\|\cdot\|_{L^{k}(\Omega)}$ respectively, and $\|\cdot\|_{0,2}$ is also used to denote $\|\cdot\|_{L^{2}(\Omega)}$. Let $\mathcal{T}_{h}$ be a family of quasi-uniform triangulations of domain $\Omega$, and $\mathcal{E}_{h}$ be a collection of edges, then the global mesh dependent semi-norm, norm and inner product are defined below

$$
\begin{aligned}
|v|_{j, p, h} & =\left(\sum_{K \in \mathcal{T}_{h}}|v|_{j, p, K}^{p}\right)^{1 / p}, \\
\|v\|_{j, p, h} & =\left(\sum_{K \in \mathcal{T}_{h}}\|v\|_{j, p, K}^{p}\right)^{1 / p}, \\
(w, v)_{h} & =\sum_{K \in \mathcal{T}_{h}} \int_{K} w(x) v(x) \mathrm{d} x .
\end{aligned}
$$

Define $L_{0}^{2}(\Omega)$ as the mean zero functions in $L^{2}(\Omega)$. For $\Phi \in L_{0}^{2}(\Omega)$, let $u:=$ $-\Delta^{-1} \Phi \in H^{1}(\Omega) \cap L_{0}^{2}(\Omega)$ such that

$$
\begin{aligned}
-\Delta u & =\Phi & & \text { in } \Omega, \\
\frac{\partial u}{\partial n} & =0 & & \text { on } \partial \Omega .
\end{aligned}
$$

Then we have

$$
-\left(\nabla \Delta^{-1} \Phi, \nabla v\right)=(\Phi, v) \quad \text { in } \Omega \quad \forall v \in H^{1}(\Omega) \cap L_{0}^{2}(\Omega) .
$$

For $v \in L_{0}^{2}(\Omega)$ and $\Phi \in L_{0}^{2}(\Omega)$, define the continuous $H^{-1}$ inner product by

$$
(\Phi, v)_{H^{-1}}:=\left(\nabla \Delta^{-1} \Phi, \nabla \Delta^{-1} v\right)=\left(\Phi,-\Delta^{-1} v\right)=\left(v,-\Delta^{-1} \Phi\right) .
$$

As in $[12,18,21,22,26,27]$, we made the following assumptions on the initial condition. These assumptions were used to derive the a priori estimates for the solution of problem (1.1)-(1.4).

General Assumption (GA)

(1) Assume that $m_{0} \in(-1,1)$ where

$$
m_{0}:=\frac{1}{|\Omega|} \int_{\Omega} u_{0}(x) \mathrm{d} x .
$$

(2) There exists a nonnegative constant $\sigma_{1}$ such that

$$
J_{\epsilon}\left(u_{0}\right) \leq C \epsilon^{-2 \sigma_{1}} .
$$


(3) There exist nonnegative constants $\sigma_{2}, \sigma_{3}$ and $\sigma_{4}$ such that

$$
\left\|-\epsilon \Delta u_{0}+\epsilon^{-1} f\left(u_{0}\right)\right\|_{H^{\ell}} \leq C \epsilon^{-\sigma_{2+\ell}} \quad \ell=0,1,2 .
$$

Under the above assumptions, the following a priori estimates of the solution were proved in $[18,21,22,26]$.

THEOREM 2.1. The solution $u$ of problem (1.1)-(1.4) satisfies the following energy estimate:

$$
\underset{t \in[0, T]}{\operatorname{ess} \sup _{t}}\left(\frac{\epsilon}{2}\|\nabla u\|_{L^{2}}^{2}+\frac{1}{\epsilon}\|F(u)\|_{L^{1}}\right)+\int_{0}^{T}\left\|u_{t}(s)\right\|_{H^{-1}}^{2} \mathrm{~d} s \leq J_{\epsilon}\left(u_{0}\right) .
$$

Moreover, suppose that $G A$ (1)-(3) hold, $u_{0} \in H^{4}(\Omega)$ and $\partial \Omega \in C^{2,1}$, then $u$ satisfies the additional estimates:

$$
\begin{aligned}
& \frac{1}{|\Omega|} \int_{\Omega} u(x, t) \mathrm{d} x=m_{0} \quad \forall t \geq 0 \\
& \text { ess sup }\|\Delta u\|_{L^{2}} \leq C \epsilon^{-\max \left\{\sigma_{1}+\frac{5}{2}, \sigma_{3}+1\right\}}, \\
& \quad \text { ess sup }\|\nabla \Delta u\|_{L^{2}} \leq C \epsilon^{-\max \left\{\sigma_{1}+\frac{5}{2}, \sigma_{3}+1\right\}}, \\
& \quad t \in[0, T] \\
& \epsilon \int_{0}^{T}\left\|\Delta u_{t}\right\|_{L^{2}}^{2} \mathrm{~d} s+\underset{t \in[0, T]}{\operatorname{ess} \sup _{t}\left\|u_{t}\right\|_{L^{2}}^{2} \leq C \epsilon^{-\max \left\{2 \sigma_{1}+\frac{13}{2}, 2 \sigma_{3}+\frac{7}{2}, 2 \sigma_{2}+4,2 \sigma_{4}\right\}} .}
\end{aligned}
$$

Furthermore, if there exists $\sigma_{5}>0$ such that

$$
\lim _{s \rightarrow 0^{+}}\left\|\nabla u_{t}(s)\right\|_{L^{2}} \leq C \epsilon^{-\sigma_{5}}
$$

then there hold

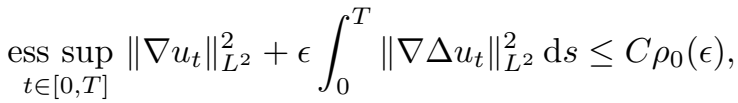

$$
\begin{aligned}
& \int_{0}^{T}\left\|u_{t t}\right\|_{H^{-1}}^{2} \mathrm{~d} s \leq C \rho_{1}(\epsilon)
\end{aligned}
$$

where

$$
\begin{aligned}
\rho_{0}(\epsilon):= & \epsilon^{-\frac{1}{2} \max \left\{2 \sigma_{1}+5,2 \sigma_{3}+2\right\}-\max \left\{2 \sigma_{1}+\frac{13}{2}, 2 \sigma_{3}+\frac{7}{2}, 2 \sigma_{2}+4\right\}}+\epsilon^{-2 \sigma_{5}} \\
& \quad+\epsilon^{-\max \left\{2 \sigma_{1}+7,2 \sigma_{3}+4\right\}} \\
\rho_{1}(\epsilon):= & \epsilon \rho_{0}(\epsilon) .
\end{aligned}
$$

Besides, an extra a priori estimates of solution $u$ is needed in this paper.

THEOREM 2.2. Under the assumptions of Theorem 2.1 and if there exists $\sigma_{6}>0$ such that

$$
\left\|\Delta u_{t}(0)\right\|_{L^{2}} \leq C \epsilon^{-\sigma_{6}}
$$

then there hold

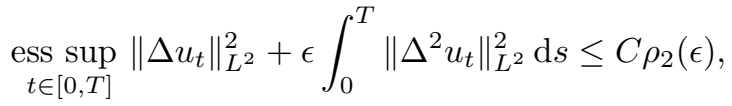

$$
\begin{aligned}
& \underset{t \in[0, T]}{\operatorname{ess} \sup } \epsilon\left\|\Delta u_{t}\right\|_{L^{2}}^{2}+\int_{0}^{T}\left\|u_{t t}\right\|_{L^{2}}^{2} \mathrm{~d} s \leq C \rho_{3}(\epsilon),
\end{aligned}
$$


where

$$
\begin{aligned}
\rho_{2}(\epsilon):= & \epsilon^{-\max \left\{2 \sigma_{1}+\frac{13}{2}, 2 \sigma_{3}+\frac{7}{2}, 2 \sigma_{2}+4,2 \sigma_{4}\right\}-\max \left\{2 \sigma_{1}+5,2 \sigma_{3}+2\right\}-3} \\
& +\epsilon^{-\max \left\{\sigma_{1}+\frac{5}{2}, \sigma_{3}+1\right\}-3} \rho_{0}(\epsilon)+\epsilon^{-2 \sigma_{6}}, \\
\rho_{3}(\epsilon):= & \epsilon \rho_{2}(\epsilon) .
\end{aligned}
$$

Proof. Using the Gagliardo-Nirenberg inequalities [1] in two-dimensional space, we have

$$
\|\nabla u\|_{L^{\infty}} \leq C\left(\|\nabla \Delta u\|_{L^{2}}^{\frac{1}{2}}\|u\|_{L^{\infty}}^{\frac{1}{2}}+\|u\|_{L^{\infty}}\right) \leq C \epsilon^{-\frac{1}{2} \max \left\{\sigma_{1}+\frac{5}{2}, \sigma_{3}+1\right\}} .
$$

Since $f^{\prime}(u)=3 u^{2}-1$, using Sobolev embedding theorem [1], (2.3), (2.5), (2.6), (2.7) and (2.9), we have

$$
\begin{aligned}
& \int_{0}^{T}\left\|\Delta\left(f^{\prime}(u) u_{t}\right)\right\|_{L^{2}}^{2} \mathrm{~d} s \\
= & \int_{0}^{T}\left\|6 u u_{t} \Delta u+12 u \nabla u \cdot \nabla u_{t}+6 u_{t} \nabla u \cdot \nabla u+\left(3 u^{2}-1\right) \Delta u_{t}\right\|_{L^{2}}^{2} \mathrm{~d} s \\
\leq & C \int_{0}^{T}\|\Delta u\|_{L^{2}}^{2}\left\|u_{t}\right\|_{L^{\infty}}^{2} \mathrm{~d} s+C \int_{0}^{T}\|\nabla u\|_{L^{\infty}}^{2}\left\|\nabla u_{t}\right\|_{L^{2}}^{2} \mathrm{~d} s \\
& +C \int_{0}^{T}\|\nabla u\|_{L^{\infty}}^{4}\left\|u_{t}\right\|_{L^{2}}^{2} \mathrm{~d} s+C \int_{0}^{T}\left\|\Delta u_{t}\right\|_{L^{2}}^{2} \mathrm{~d} s \\
\leq & C\|\Delta u\|_{L^{\infty}\left(L^{2}\right)}^{2} \int_{0}^{T}\left\|u_{t}\right\|_{H^{2}}^{2} \mathrm{~d} s+C\left\|\nabla u_{t}\right\|_{L^{\infty}\left(L^{2}\right)}^{2}\|\nabla u\|_{L^{\infty}\left(L^{\infty}\right)}^{2} \\
& +C\|\nabla u\|_{L^{\infty}\left(L^{\infty}\right)}^{4}\left\|u_{t}\right\|_{L^{\infty}\left(L^{2}\right)}^{2}+C \int_{0}^{T}\left\|\Delta u_{t}\right\|_{L^{2}}^{2} \mathrm{~d} s \\
\leq & C \epsilon^{-\max \left\{2 \sigma_{1}+\frac{13}{2}, 2 \sigma_{3}+\frac{7}{2}, 2 \sigma_{2}+4,2 \sigma_{4}\right\}-\max \left\{2 \sigma_{1}+5,2 \sigma_{3}+2\right\}-1} \\
& +C \epsilon^{-\max \left\{\sigma_{1}+\frac{5}{2}, \sigma_{3}+1\right\}} \rho_{0}(\epsilon) \\
& +C \epsilon^{-\max \left\{2 \sigma_{1}+\frac{13}{2}, 2 \sigma_{3}+\frac{7}{2}, 2 \sigma_{2}+4,2 \sigma_{4}\right\}-\max \left\{2 \sigma_{1}+5,2 \sigma_{3}+2\right\}} \\
& +C \epsilon^{-\max \left\{2 \sigma_{1}+\frac{13}{2}, 2 \sigma_{3}+\frac{7}{2}, 2 \sigma_{2}+4,2 \sigma_{4}\right\}-1} \\
\leq & C \epsilon^{-\max \left\{2 \sigma_{1}+\frac{13}{2}, 2 \sigma_{3}+\frac{7}{2}, 2 \sigma_{2}+4,2 \sigma_{4}\right\}-\max \left\{2 \sigma_{1}+5,2 \sigma_{3}+2\right\}} \\
& +C \epsilon^{-\max \left\{\sigma_{1}+\frac{5}{2}, \sigma_{3}+1\right\}} \rho_{0}(\epsilon) .
\end{aligned}
$$

Taking the derivative with respect to $t$ on both sides of (1.1), we get

$$
u_{t t}+\epsilon \Delta^{2} u_{t}-\frac{1}{\epsilon} \Delta\left(f^{\prime}(u) u_{t}\right)=0 .
$$

Testing (2.16) with $\Delta^{2} u_{t}$, and taking the integral over $(0, T)$, we obtain

$$
\begin{aligned}
& \frac{1}{2}\left\|\Delta u_{t}(T)\right\|_{L^{2}}^{2}+\epsilon \int_{0}^{T}\left\|\Delta^{2} u_{t}\right\|_{L^{2}}^{2} \mathrm{~d} s \\
= & \frac{1}{\epsilon} \int_{0}^{T}\left(\Delta\left(f^{\prime}(u) u_{t}\right), \Delta^{2} u_{t}\right) \mathrm{d} s+\frac{1}{2}\left\|\Delta u_{t}(0)\right\|_{L^{2}}^{2} \\
\leq & \frac{C}{\epsilon^{3}} \int_{0}^{T}\left\|\Delta\left(f^{\prime}(u) u_{t}\right)\right\|_{L^{2}}^{2} \mathrm{~d} s+\frac{\epsilon}{2} \int_{0}^{T}\left\|\Delta^{2} u_{t}\right\|_{L^{2}}^{2} \mathrm{~d} s+C \epsilon^{-2 \sigma_{6}} .
\end{aligned}
$$


Then (2.12) is obtained by (2.15).

Next we bound (2.13). Testing (2.16) with $u_{t t}$, taking the integral over $(0, T)$, and using (2.17), we obtain

$$
\begin{aligned}
& \int_{0}^{T}\left\|u_{t t}\right\|_{L^{2}}^{2} \mathrm{~d} s+\frac{\epsilon}{2}\left\|\Delta u_{t}(T)\right\|_{L^{2}}^{2} \\
\leq & \frac{\epsilon}{2}\left\|\Delta u_{t}(0)\right\|_{L^{2}}^{2}+\frac{C}{\epsilon^{2}} \int_{0}^{T}\left\|\Delta\left(f^{\prime}(u) u_{t}\right)\right\|_{L^{2}}^{2} \mathrm{~d} s+\frac{1}{2} \int_{0}^{T}\left\|u_{t t}\right\|_{L^{2}}^{2} \mathrm{~d} s .
\end{aligned}
$$

Then (2.13) is obtained by (2.15).

The next lemma gives an $\epsilon$-independent lower bound for the principal eigenvalue of the linearized Cahn-Hilliard operator $\mathcal{L}_{C H}$ defined below. The proof of this lemma can be found in [12].

Lemma 2.3. Suppose that GA (1)-(3) hold. Given a smooth initial curve/surface $\Gamma_{0}$, let $u_{0}$ be a smooth function satisfying $\Gamma_{0}=\left\{x \in \Omega ; u_{0}(x)=0\right\}$ and some profile described in [12]. Let $u$ be the solution to problem (1.1)-(1.4). Define $\mathcal{L}_{C H}$ as

$$
\mathcal{L}_{C H}:=\Delta\left(\epsilon \Delta-\frac{1}{\epsilon} f^{\prime}(u) I\right) .
$$

Then there exists $0<\epsilon_{0} \ll 1$ and a positive constant $C_{0}$ such that the principle eigenvalue of the linearized Cahn-Hilliard operator $\mathcal{L}_{C H}$ satisfies

$$
\lambda_{C H}:=\inf _{\substack{0 \neq \psi \in H^{1}(\Omega) \\ \Delta w=\psi}} \frac{\epsilon\|\nabla \psi\|_{L^{2}}^{2}+\frac{1}{\epsilon}\left(f^{\prime}(u) \psi, \psi\right)}{\|\nabla w\|_{L^{2}}^{2}} \geq-C_{0}
$$

for $t \in[0, T]$ and $\epsilon \in\left(0, \epsilon_{0}\right)$.

3. Fully Discrete Approximation. In this section, the backward Euler is used for time stepping, and the Morley finite element discretization is used for space discretization.

3.1. Morley finite element space. Define the Morley finite element spaces $S^{h}$ below $[8,10,14]$ :

$$
\begin{aligned}
S^{h}:=\left\{v_{h} \in L^{\infty}(\Omega): v_{h} \in P_{2}(K), v_{h}\right. \text { is continuous at the vertices of all triangles, } & \left.\frac{\partial v_{h}}{\partial n} \text { is continuous at the midpoints of interelement edges of triangles }\right\} .
\end{aligned}
$$

We use the following notation

$$
H_{E}^{j}(\Omega):=\left\{v \in H^{j}(\Omega): \frac{\partial v}{\partial n}=0 \text { on } \partial \Omega\right\} \quad j=1,2,3 .
$$

Corresponding to $H_{E}^{j}(\Omega)$, define $S_{E}^{h}$ as a subspace of $S^{h}$ below:

$$
S_{E}^{h}:=\left\{v_{h} \in S^{h}: \frac{\partial v_{h}}{\partial n}=0 \text { at the midpoints of the edges on } \partial \Omega\right\} .
$$

We also define $\stackrel{\circ}{H}_{E}^{j}(\Omega)=H_{E}^{j}(\Omega) \cap L_{0}^{2}(\Omega), j=1,2,3$, and $\stackrel{\circ}{S}_{E}^{h}=S_{E}^{h} \cap L_{0}^{2}(\Omega)$, where $L_{0}^{2}(\Omega)$ denotes the set of mean zero functions. 
The enriching operator $\widetilde{E}_{h}$ is restated $[7,8,10]$. Let $\widetilde{S}_{E}^{h}$ be the Hsieh-CloughTocher macro element space, which is an enriched space of the Morley finite element space $S_{E}^{h}$. Let $p$ and $m$ be the internal vertices and midpoints of triangles $\mathcal{T}_{h}$. Define $\widetilde{E}_{h}: S_{E}^{h} \rightarrow \widetilde{S}_{E}^{h}$ by

$$
\begin{aligned}
\left(\widetilde{E}_{h} v\right)(p) & =v(p), \\
\frac{\partial\left(\widetilde{E}_{h} v\right)}{\partial n}(m) & =\frac{\partial v}{\partial n}(m), \\
\left(\partial^{\beta}\left(\widetilde{E}_{h} v\right)\right)(p) & =\text { average of }\left(\partial^{\beta} v_{i}\right)(p) \quad|\beta|=1,
\end{aligned}
$$

where $v_{i}=\left.v\right|_{T_{i}}$ and triangle $T_{i}$ contains $p$ as a vertex.

Define the interpolation operator $I_{h}: H_{E}^{2}(\Omega) \rightarrow S_{E}^{h}$ such that

$$
\begin{aligned}
\left(I_{h} v\right)(p) & =v(p), \\
\frac{\partial\left(I_{h} v\right)}{\partial n}(m) & =\frac{1}{|e|} \int_{e} \frac{\partial v}{\partial n} \mathrm{~d} S
\end{aligned}
$$

where $p$ ranges over the internal vertices of all the triangles $T$, and $m$ ranges over the midpoints of all the edges $e$. It can be proved that $[7,8,10,14]$

$$
\begin{aligned}
\left|v-I_{h} v\right|_{j, p, K} & \leq C h^{3-j}|v|_{3, p, K} & \forall K \in \mathcal{T}_{h}, & \forall v \in H^{3}(K), \quad j=0,1,2, \\
\left\|\widetilde{E}_{h} v-v\right\|_{j, 2, h} & \leq C h^{2-j}|v|_{2,2, h} & \forall v \in S_{E}^{h}, & j=0,1,2 .
\end{aligned}
$$

Notice that $\widetilde{E}_{h}$ and $I_{h}$ cannot preserve the mean zero functions. Let $\widetilde{S}_{E}^{h}:=$ $\widetilde{S}_{E}^{h} \cap L_{0}^{2}(\Omega)$. Define $\stackrel{\circ}{E}_{h}: \stackrel{\circ}{S}_{E}^{h} \mapsto \stackrel{\circ}{S}_{E}^{h}$ such that

$$
\widetilde{\widetilde{E}}_{h} v=\widetilde{E}_{h} v-\frac{1}{|\Omega|} \int_{\Omega} \widetilde{E}_{h} v \mathrm{~d} x .
$$

Using (3.2), we have

$$
\int_{\Omega} \widetilde{E}_{h} v \mathrm{~d} x=\left(\widetilde{E}_{h} v-v, 1\right) \leq|\Omega|^{1 / 2}\left\|\widetilde{E}_{h} v-v\right\|_{0,2} \leq C h^{2}|v|_{2,2, h} \quad \forall v \in \stackrel{\leftrightarrow}{S}_{E}^{h} .
$$

Then

$$
\left\|\stackrel{\odot}{E}_{h} v-v\right\|_{j, 2, h} \leq C h^{2-j}|v|_{2,2, h} \quad \forall v \in \stackrel{\circ}{S}_{E}^{h}, \quad j=0,1,2 .
$$

Finally the following spaces are needed

$$
\begin{array}{ll}
H^{3, h}(\Omega)=S^{h} \oplus H^{3}(\Omega), & H_{E}^{3, h}(\Omega)=S_{E}^{h} \oplus H_{E}^{3}(\Omega), \\
H^{2, h}(\Omega)=S^{h} \oplus H^{2}(\Omega), & H_{E}^{2, h}(\Omega)=S_{E}^{h} \oplus H_{E}^{2}(\Omega), \\
H^{1, h}(\Omega)=S^{h} \oplus H^{1}(\Omega), & H_{E}^{1, h}(\Omega)=S_{E}^{h} \oplus H_{E}^{1}(\Omega),
\end{array}
$$

where, for instance,

$$
S_{E}^{h} \oplus H_{E}^{3}(\Omega):=\left\{u+v: u \in S_{E}^{h} \text { and } v \in H_{E}^{3}(\Omega)\right\} .
$$


3.2. Formulation. The weak form of $(1.1)-(1.4)$ is to seek $u(\cdot, t) \in H_{E}^{2}(\Omega)$ such that

$$
\begin{aligned}
\left(u_{t}, v\right)+\epsilon a(u, v)+\frac{1}{\epsilon}(\nabla f(u), \nabla v) & =0 \quad \forall v \in H_{E}^{2}(\Omega), \\
u(\cdot, 0) & =u_{0} \in H_{E}^{2}(\Omega)
\end{aligned}
$$

where the bilinear form $a(\cdot, \cdot)$ is defined as

$$
a(u, v):=\int_{\Omega} \Delta u \Delta v+\left(\frac{\partial^{2} u}{\partial x \partial y} \frac{\partial^{2} v}{\partial x \partial y}-\frac{1}{2} \frac{\partial^{2} u}{\partial x^{2}} \frac{\partial^{2} v}{\partial y^{2}}-\frac{1}{2} \frac{\partial^{2} u}{\partial y^{2}} \frac{\partial^{2} v}{\partial x^{2}}\right) \mathrm{d} x \mathrm{~d} y
$$

with Poisson's ratio $\frac{1}{2}$.

Next define the discrete bilinear form

$$
a_{h}(u, v):=\sum_{K \in \mathcal{T}_{h}} \int_{K} \Delta u \Delta v+\left(\frac{\partial^{2} u}{\partial x \partial y} \frac{\partial^{2} v}{\partial x \partial y}-\frac{1}{2} \frac{\partial^{2} u}{\partial x^{2}} \frac{\partial^{2} v}{\partial y^{2}}-\frac{1}{2} \frac{\partial^{2} u}{\partial y^{2}} \frac{\partial^{2} v}{\partial x^{2}}\right) \mathrm{d} x \mathrm{~d} y
$$

Based on the bilinear form (3.8), a fully discrete Galerkin method is to seek $u_{h}^{n} \in S_{E}^{h}$ such that

$$
\begin{aligned}
\left(d_{t} u_{h}^{n}, v_{h}\right)+\epsilon a_{h}\left(u_{h}^{n}, v_{h}\right)+\frac{1}{\epsilon}\left(\nabla f\left(u_{h}^{n}\right), \nabla v_{h}\right)_{h} & =0 \quad \forall v_{h} \in S_{E}^{h}, \\
u_{h}^{0} & =u_{0}^{h} \in S_{E}^{h},
\end{aligned}
$$

where the difference operator $d_{t} u_{h}^{n}:=\frac{u_{h}^{n}-u_{h}^{n-1}}{k}$ and $u_{0}^{h}:=P_{h} u\left(t_{0}\right)$, where the operator $P_{h}$ is defined below.

3.3. Elliptic operator $P_{h}$. We define

$$
R:=\left\{v \in H_{E}^{2}(\Omega): \Delta v \in H_{E}^{2}(\Omega)\right\} .
$$

Then $\forall v \in R$, define the elliptic operator $P_{h}$ (cf. [14]) by seeking $P_{h} v \in S_{E}^{h}$ such that

$$
\tilde{b}_{h}\left(P_{h} v, w\right):=\left(\epsilon \Delta^{2} v-\frac{1}{\epsilon} \nabla \cdot\left(f^{\prime}(u) \nabla v\right)+\alpha v, w\right) \quad \forall w \in S_{E}^{h},
$$

where

$$
\tilde{b}_{h}(v, w):=\epsilon a_{h}(v, w)+\frac{1}{\epsilon}\left(f^{\prime}(u) \nabla v, \nabla w\right)_{h}+\alpha(v, w)
$$

and $\alpha$ should be chosen as $\alpha=\alpha_{0} \epsilon^{-3}$ to guarantee the coercivity of $\tilde{b}_{h}(\cdot, \cdot)$. More precisely, first we cite some lemmas in [14], which will be used in this paper.

LEMMA 3.1 (Lemma 2.3 in [14]). Let $w, z \in H_{E}^{2, h}(\Omega)$, then

$$
\left|\sum_{K \in \mathcal{T}_{h}} \int_{\partial K} \frac{\partial w}{\partial n} z \mathrm{~d} S\right| \leq C h\left(h\|w\|_{2,2, h}\|z\|_{2,2, h}+\|w\|_{1,2, h}\|z\|_{2,2, h}+\|w\|_{2,2, h}\|z\|_{1,2, h}\right) .
$$

LEMMA 3.2 (Lemma 2.5 in [14]). Let $z \in H^{2, h}(\Omega)$ and $w \in H_{E}^{2}(\Omega) \cap H^{3}(\Omega)$, and define $B_{h}(w, z)$ by

$$
B_{h}(w, z)=\sum_{K \in \mathcal{T}_{h}} \int_{\partial K}\left(\Delta w \frac{\partial z}{\partial n}+\frac{1}{2} \frac{\partial^{2} w}{\partial n \partial s}-\frac{1}{2} \frac{\partial^{2} w}{\partial s^{2}} \frac{\partial z}{\partial n}\right) \mathrm{d} S
$$

then we have

$$
\left|B_{h}(w, z)\right| \leq C h|w|_{3,2, h}|z|_{2,2, h}
$$


For any $w \in S_{E}^{h}$, using Lemma 3.1 and the inverse inequality, we have

$$
\begin{aligned}
|w|_{1,2, h}^{2} & \leq|w|_{2,2, h}\|w\|_{0,2}+\left|\sum_{K \in \mathcal{T}_{h}} \int_{\partial K} \frac{\partial w}{\partial n} z \mathrm{~d} S\right| \leq C\|w\|_{2,2, h}\|w\|_{0,2} \\
& \leq C\left(|w|_{2,2, h}\|w\|_{0,2}+|w|_{1,2, h}\|w\|_{0,2}+\|w\|_{0,2}^{2}\right) .
\end{aligned}
$$

The kick-back argument gives

$$
|w|_{1,2, h}^{2} \leq C\left(|w|_{2,2, h}\|w\|_{0,2}+\|w\|_{0,2}^{2}\right) .
$$

Hence,

$$
\begin{aligned}
\tilde{b}_{h}(w, w) & =\epsilon a_{h}(w, w)+\frac{1}{\epsilon}\left(f^{\prime}(u) \nabla w, \nabla w\right)+\frac{\alpha_{0}}{\epsilon^{3}}(w, w) \\
& \geq \frac{1}{\epsilon^{3}}\left(\frac{\epsilon^{4}}{2}|w|_{2,2, h}^{2}-C \epsilon^{2}|w|_{1,2, h}^{2}+\alpha_{0}\|w\|_{0,2}^{2}\right) \\
& \geq \frac{1}{\epsilon^{3}}\left(\frac{\epsilon^{4}}{4}|w|_{2,2, h}^{2}+\left(\alpha_{0}-C\right)\|w\|_{0,2}^{2}\right),
\end{aligned}
$$

which implies the coercivity of $\tilde{b}_{h}(\cdot, \cdot)$ when $\alpha_{0}$ is large enough but independent of $\epsilon$.

Next we give the properties of $P_{h}$. Define $b_{h}(\cdot, \cdot):=\epsilon^{3} \tilde{b}_{h}(\cdot, \cdot)$ and a norm

$$
\|v\|_{2,2, h}^{2}:=\epsilon^{4}|v|_{2,2, h}^{2}+\epsilon^{2}|v|_{1,2, h}^{2}+\|v\|_{0,2}^{2},
$$

Lemma 3.3. Consider the following problems:

$$
\begin{aligned}
b_{h}(v, \eta) & =F_{h}(\eta) \quad \forall \eta \in H_{E}^{2}(\Omega), \\
b_{h}\left(v_{h}, \chi\right) & =\widetilde{F}_{h}(\chi) \quad \forall \chi \in S_{E}^{h} .
\end{aligned}
$$

Then we have

$$
\begin{aligned}
& \left\|v-v_{h}\right\|_{2,2, h} \\
\leq & C h\left\{(\epsilon+h)^{2}|v|_{3,2}+|v|_{1,2}+\sup _{\chi \in S_{E}^{h}} \frac{F_{h}\left(\widetilde{E}_{h} \chi\right)-\widetilde{F}_{h}(\chi)+\alpha_{0}\left(v, \chi-\widetilde{E}_{h} \chi\right)}{\|\chi\|_{2,2, h}}\right\} .
\end{aligned}
$$

Proof. Using (3.14) and the Strang Lemma, we have

$$
\begin{aligned}
& \left\|v-v_{h}\right\|_{2,2, h} \\
\leq & C\left(\inf _{\psi \in S_{E}^{h}}\|v-\psi\|_{2,2, h}+\sup _{\chi \in S_{E}^{h}} \frac{b_{h}(v, \chi)-\widetilde{F}_{h}(\chi)}{\|\chi\|_{2,2, h}}\right) \\
\leq & C\left(\inf _{\psi \in S_{E}^{h}}\|v-\psi\|_{2,2, h}+\sup _{\chi \in S_{E}^{h}} \frac{b_{h}\left(v, \chi-\widetilde{E}_{h} \chi\right)+b_{h}\left(v, \widetilde{E}_{h} \chi\right)-\widetilde{F}_{h}(\chi)}{\|\chi\|_{2,2, h}}\right) \\
\leq & C\left(\inf _{\psi \in S_{E}^{h}}\|v-\psi\|_{2,2, h}+\sup _{\chi \in S_{E}^{h}} \frac{b_{h}\left(v, \chi-\widetilde{E}_{h} \chi\right)+F_{h}\left(\widetilde{E}_{h} \chi\right)-\widetilde{F}_{h}(\chi)}{\|\chi\|_{2,2, h}}\right) .
\end{aligned}
$$

Using Lemma 3.2 and (3.2), we have

$$
\begin{aligned}
b_{h}\left(v, \chi-\widetilde{E}_{h} \chi\right) & =\epsilon^{4} a_{h}\left(v, \chi-\widetilde{E}_{h} \chi\right)+\epsilon^{2}\left(f^{\prime}(u) \nabla v, \nabla\left(\chi-\widetilde{E}_{h} \chi\right)\right)+\left(\alpha_{0} v, \chi-\widetilde{E}_{h} \chi\right) \\
& \leq C h\left(\epsilon^{4}|v|_{3,2}|\chi|_{2,2, h}+\epsilon^{2}|v|_{1,2}|\chi|_{2,2, h}\right)+\left(\alpha_{0} v, \chi-\widetilde{E}_{h} \chi\right) \\
& \leq C h\left(\epsilon^{2}|v|_{3,2}+|v|_{1,2}\right)\||| \mid\|_{2,2, h}+\left(\alpha_{0} v, \chi-\widetilde{E}_{h} \chi\right)
\end{aligned}
$$


Then we obtain the desired bound (3.17) by the approximation properties of Morley interpolation operator (3.1).

Theorem 3.4. Suppose u solves the Cahn-Hilliard equation (1.1) - (1.3), then we have

$$
\begin{aligned}
& \epsilon^{2}\left|u-P_{h} u\right|_{2,2, h}+\epsilon\left|u-P_{h} u\right|_{1,2, h}+\left\|u-P_{h} u\right\|_{0,2} \\
\leq & C h\left((\epsilon+h)^{2}|u|_{3,2}+|u|_{1,2}+\epsilon h\left\|u_{t}\right\|_{0,2}\right), \\
& \epsilon^{2}\left|u_{t}-\left(P_{h} u\right)_{t}\right|_{2,2, h}+\epsilon\left|u_{t}-\left(P_{h} u\right)_{t}\right|_{1,2, h}+\left\|u_{t}-\left(P_{h} u\right)_{t}\right\|_{0,2} \\
\leq & C h\left\{(\epsilon+h)^{2}\left|u_{t}\right|_{3,2}+\left|u_{t}\right|_{1,2}+\epsilon h\left\|u_{t t}\right\|_{0,2}+\left\|u_{t} \nabla u\right\|_{0,2}\right. \\
& \left.+\epsilon^{-1}|\ln h|^{1 / 2}\left\|u_{t}\right\|_{0,2}\left((\epsilon+h)^{2}|u|_{3,2}+|u|_{1,2}+\epsilon h\left\|u_{t}\right\|_{0,2}\right)\right\} .
\end{aligned}
$$

Proof. Taking $v=u$ and $v_{h}=P_{h} u$ in Lemma 3.3, and noticing that

$$
F_{h}(\psi)=\tilde{F}_{h}(\psi)=\left(\epsilon^{4} \Delta^{2} u-\epsilon^{2} \Delta f(u)+\alpha_{0} u, \psi\right)=\left(\epsilon^{3} u_{t}+\alpha_{0} u, \psi\right),
$$

we obtain the bound (3.18) from (3.2) and (3.17).

Taking $v=u_{t}$ and $v_{h}=\left(P_{h} u\right)_{t}$, we have

$$
\begin{aligned}
& F_{h}(\psi)=\left(\epsilon^{4} \Delta^{2} u_{t}-\epsilon^{2} \Delta f(u)_{t}+\alpha_{0} u_{t}, \psi\right)-\left(\epsilon^{2} f^{\prime \prime}(u) u_{t} \nabla u, \nabla \psi\right)_{h}, \\
& \widetilde{F}_{h}(\psi)=\left(\epsilon^{4} \Delta^{2} u_{t}-\epsilon^{2} \Delta f(u)_{t}+\alpha_{0} u_{t}, \psi\right)-\left(\epsilon^{2} f^{\prime \prime}(u) u_{t} \nabla P_{h} u, \nabla \psi\right)_{h} .
\end{aligned}
$$

Then we get

$$
\begin{aligned}
& F_{h}\left(\widetilde{E}_{h} \chi\right)-\widetilde{F}(\chi)+\alpha_{0}\left(u_{t}, \chi-\widetilde{E}_{h} \chi\right) \\
= & \left(\epsilon^{4} \Delta^{2} u_{t}-\epsilon^{2} \Delta f(u)_{t}, \widetilde{E}_{h} \chi-\chi\right) \\
& -\left(\epsilon^{2} f^{\prime \prime}(u) u_{t} \nabla u, \nabla \widetilde{E}_{h} \chi-\nabla \chi\right)-\left(\epsilon^{2} f^{\prime \prime}(u) u_{t} \nabla\left(u-P_{h} u\right), \nabla \chi\right) \\
\leq & \epsilon^{3} h^{2}\left\|u_{t t}\right\|_{0,2}|\chi|_{2,2, h}+C \epsilon^{2} h\left\|u_{t} \nabla u\right\|_{0,2}|\chi|_{2,2, h}+C \epsilon^{2}\left\|u_{t}\right\|_{0,2}\|\nabla \chi\|_{0, \infty}\left|u-P_{h} u\right|_{1,2, h} \\
\leq & C h\left\{\epsilon h\left\|u_{t t}\right\|_{0,2}+\left\|u_{t} \nabla u\right\|_{0,2}\right. \\
& \left.+\epsilon^{-1}|\ln h|^{1 / 2}\left\|u_{t}\right\|_{0,2}\left((\epsilon+h)^{2}|u|_{3,2}+|u|_{1,2}+\epsilon h\left\|u_{t}\right\|_{0,2}\right)\right\}\|\chi\|_{2,2, h},
\end{aligned}
$$

where we use the discrete Sobolev inequality and the fact that $\nabla \chi$ belongs to the Crouzeix-Raviar finite element space [9]. This implies the bound (3.19).

Combining with the a priori estimates of the bounds given in Section 2, we have the following theorem.

Theorem 3.5. Assume $h \leq C \epsilon$, then there hold

$$
\begin{aligned}
& \epsilon^{4}\left|u-P_{h} u\right|_{2,2, h}^{2}+\epsilon^{2}\left|u-P_{h} u\right|_{1,2, h}^{2}+\left\|u-P_{h} u\right\|_{0,2}^{2} \leq C h^{2} \rho_{4}(\epsilon), \\
& \quad \int_{0}^{T} \epsilon^{4}\left|u_{t}-\left(P_{h} u\right)_{t}\right|_{2,2, h}^{2}+\epsilon^{2}\left|u_{t}-\left(P_{h} u\right)_{t}\right|_{1,2, h}^{2}+\left\|u_{t}-\left(P_{h} u\right)_{t}\right\|_{0,2}^{2} \mathrm{~d} s \\
& \leq C h^{2} \epsilon^{4} \rho_{3}(\epsilon)+C h^{2}|\ln h| \rho_{5}(\epsilon),
\end{aligned}
$$

where

$$
\begin{aligned}
& \rho_{4}(\epsilon):=\epsilon^{-\max \left\{2 \sigma_{1}+\frac{13}{2}, 2 \sigma_{3}+\frac{7}{2}, 2 \sigma_{2}+4,2 \sigma_{4}\right\}+4}, \\
& \rho_{5}(\epsilon):=\epsilon^{-2 \max \left\{2 \sigma_{1}+\frac{13}{2}, 2 \sigma_{3}+\frac{7}{2}, 2 \sigma_{2}+4,2 \sigma_{4}\right\}+2} .
\end{aligned}
$$


Proof. Using (2.3), (2.6) and (2.7), we have

$$
\begin{aligned}
& (\epsilon+h)^{4}|u|_{3,2}^{2}+|u|_{1,2}^{2}+\epsilon^{2} h^{2}\left\|u_{t}\right\|_{0,2}^{2} \\
\leq & C \epsilon^{-\max \left\{2 \sigma_{1}+5,2 \sigma_{3}+2\right\}+4}+C \epsilon^{-2 \sigma_{1}-1}+C \epsilon^{-\max \left\{2 \sigma_{1}+\frac{13}{2}, 2 \sigma_{3}+\frac{7}{2}, 2 \sigma_{2}+4,2 \sigma_{4}\right\}+4} \\
\leq & C \rho_{4}(\epsilon),
\end{aligned}
$$

which implies the bound (3.20) by (3.18).

Using (2.7), (2.13), (2.9) and (2.14), we obtain

$$
\begin{aligned}
& \int_{0}^{T}(\epsilon+h)^{4}\left|u_{t}\right|_{3,2}^{2}+\left|u_{t}\right|_{1,2}^{2}+\epsilon^{2} h^{2}\left\|u_{t t}\right\|_{0,2}^{2}+\left\|u_{t} \nabla u\right\|_{0,2}^{2} \mathrm{~d} s \\
\leq & C \int_{0}^{T} \epsilon^{4}\left|u_{t}\right|_{3,2}^{2}+\left|u_{t}\right|_{1,2}^{2}+\epsilon^{4}\left\|u_{t t}\right\|_{0,2}^{2}+\left\|u_{t}\right\|_{0,2}^{2}\|\nabla u\|_{0, \infty}^{2} \mathrm{~d} s \\
\leq & C \epsilon^{3} \rho_{0}(\epsilon)+C \rho_{0}(\epsilon)+C \epsilon^{4} \rho_{3}(\epsilon) \\
+ & C \epsilon^{-\max \left\{\sigma_{1}+\frac{5}{2}, \sigma_{3}+1\right\}-\max \left\{2 \sigma_{1}+\frac{13}{2}, 2 \sigma_{3}+\frac{7}{2}, 2 \sigma_{2}+4,2 \sigma_{4}\right\}} \\
\leq & C \epsilon^{4} \rho_{3}(\epsilon) .
\end{aligned}
$$

Further, using (2.7) and (3.22), we obtain

$$
\int_{0}^{T} \epsilon^{-2}\left\|u_{t}\right\|_{0,2}^{2}\left((\epsilon+h)^{2}|u|_{3,2}+|u|_{1,2}+\epsilon h\left\|u_{t}\right\|_{0,2}\right)^{2} \mathrm{~d} s \leq C \rho_{5}(\epsilon) .
$$

This implies the bound (3.21).

COROLlary 3.6. Under the condition that

$$
h \leq C \epsilon^{2} \rho_{4}^{-\frac{1}{2}}(\epsilon), \quad h \leq C \rho_{3}^{-\frac{1}{2}}(\epsilon), \quad h|\ln h|^{\frac{1}{2}} \leq C \epsilon^{2} \rho_{5}^{-\frac{1}{2}}(\epsilon),
$$

there hold

$$
\begin{aligned}
\left|P_{h} u\right|_{j, 2, h}^{2} & \leq C\left(1+|u|_{j, 2, h}^{2}\right) \quad j=0,1,2, \\
\int_{0}^{T}\left|P_{h} u\right|_{j, 2, h}^{2} \mathrm{~d} s & \leq C\left(1+\int_{0}^{T}|u|_{j, 2, h}^{2}\right) \quad j=0,1,2, \\
\left\|P_{h} u\right\|_{0, \infty} & \leq C .
\end{aligned}
$$

Proof. By the Sobolev embedding and (3.20), we have

$$
\left\|P_{h} u\right\|_{0, \infty} \leq\|u\|_{0, \infty}+\left\|u-P_{h} u\right\|_{2,2, h} \leq C+C h \epsilon^{-2} \rho_{4}^{1 / 2}(\epsilon) \leq C .
$$

The first two bounds are the direct consequences of Theorem 3.5.

4. Error Estimates. In this section, first we derive the piecewise $L^{\infty}\left(L^{2}\right)$ and $L^{2}\left(H^{2}\right)$ error bounds which depend on $\frac{1}{\epsilon}$ polynomially based on the generalized coercivity result in Theorem 4.3, and piecewise $L^{\infty}\left(H^{-1}\right)$ and $L^{2}\left(H^{1}\right)$ error bounds. Then we prove the piecewise $L^{\infty}\left(H^{2}\right)$ error bound based on the piecewise $L^{\infty}\left(L^{2}\right)$ and $L^{2}\left(H^{2}\right)$ error bounds. Finally, the $L^{\infty}\left(L^{\infty}\right)$ error bound is established.

Decompose the error

$$
u-u_{h}^{n}=\left(u-P_{h} u\right)+\left(P_{h} u-u_{h}^{n}\right):=\rho^{n}+\theta^{n} .
$$

The following two lemmas will be used in this section. 
Lemma 4.1 (Summation by parts). Suppose $\left\{a_{n}\right\}_{n=0}^{\ell}$ and $\left\{b_{n}\right\}_{n=0}^{\ell}$ are two sequences, then

$$
\sum_{n=1}^{\ell}\left(a^{n}-a^{n-1}, b^{n}\right)=\left(a^{\ell}, b^{\ell}\right)-\left(a^{0}, b^{0}\right)-\sum_{n=1}^{\ell}\left(a^{n-1}, b^{n}-b^{n-1}\right) .
$$

Lemma 4.2. Suppose $u\left(t_{n}\right)$ to be the solution of (1.1)-(1.4), and $u_{h}^{n}$ to be the solution of (3.9)-(3.10), then

$$
\rho^{n} \in \stackrel{\circ}{S}_{E}^{h}, \quad \theta^{n} \in \stackrel{\circ}{S}_{E}^{h} .
$$

Proof. Testing (1.1) with constant 1, and then taking the integration over $(0, t)$, we can obtain for any $t \geq 0$,

$$
\int_{\Omega} u(t) d x=\int_{\Omega} u(0) d x .
$$

Then choosing $v=u(t), w=1$ in (3.11), we have for any $t \geq 0$,

$$
\int_{\Omega} P_{h} u(t) \mathrm{d} x=\int_{\Omega} u(t) \mathrm{d} x
$$

Choosing $v_{h}=1$ in (3.9), then

$$
\int_{\Omega} u_{h}^{n} \mathrm{~d} x=\int_{\Omega} u_{h}^{n-1} \mathrm{~d} x=\cdots=\int_{\Omega} u_{h}^{0} \mathrm{~d} x .
$$

Therefore, if choosing $u_{h}^{0}=P_{h} u(0)$, then

$$
\begin{aligned}
\int_{\Omega} u_{h}^{n} \mathrm{~d} x & =\int_{\Omega} u_{h}^{0} \mathrm{~d} x=\int_{\Omega} P_{h} u(0) \mathrm{d} x \\
& =\int_{\Omega} u(0) \mathrm{d} x=\int_{\Omega} u\left(t_{n}\right) \mathrm{d} x=\int_{\Omega} P_{h} u\left(t_{n}\right) \mathrm{d} x .
\end{aligned}
$$

Hence, $P_{h} u\left(t_{n}\right)-u_{h}^{n} \in \stackrel{\leftrightarrow}{S}_{E}^{h}$.

4.1. Generalized coercivity result, piecewise $L^{\infty}\left(H^{-1}\right)$ and $L^{2}\left(H^{1}\right)$ error estimates. We first cite the generalized coercivity result, piecewise $L^{\infty}\left(H^{-1}\right)$ and $L^{2}\left(H^{1}\right)$ error estimates established in [27].

THEOREM 4.3 (Generalized coercivity). Suppose there exists a positive number $\gamma_{3}>0$ such that the solution $u$ of problem (1.1)-(1.4) and elliptic operator $P_{h}$ satisfy

$$
\left\|u-P_{h} u\right\|_{L^{\infty}\left((0, T) ; L^{\infty}\right)} \leq C_{1} h \epsilon^{-\gamma_{3}} .
$$

Then there exists an $\epsilon$-independent and $h$-independent constant $C>0$ such that for $\epsilon \in\left(0, \epsilon_{0}\right)$, a.e. $t \in[0, T]$, and for any $\psi \in \stackrel{\circ}{S}_{E}^{h}$,

$$
\left(\epsilon-\epsilon^{4}\right)(\nabla \psi, \nabla \psi)_{h}+\frac{1}{\epsilon}\left(f^{\prime}\left(P_{h} u(t)\right) \psi, \psi\right)_{h} \geq-C\left\|\nabla \Delta^{-1} \psi\right\|_{L^{2}}^{2}-C \epsilon^{-2 \gamma_{2}-4} h^{4},
$$

provided that $h$ satisfies the constraint

$$
h \leq\left(C_{1} C_{2}\right)^{-1} \epsilon^{\gamma_{3}+3},
$$

where $\gamma_{2}=2 \gamma_{1}+\sigma_{1}+6$ and $C_{2}$ is determined by

$$
C_{2}:=\max _{|\xi| \leq\|u\|_{L \infty}((0, T) ; L \infty)}\left|f^{\prime \prime}(\xi)\right| .
$$


REMARK 1. Thanks to the Sobolev embedding theorem and (3.20), we have

$$
\left\|u-P_{h} u\right\|_{0, \infty} \leq\left\|u-P_{h} u\right\|_{2,2, h} \leq C h \epsilon^{-2} \rho_{4}^{\frac{1}{2}}(\epsilon),
$$

which gives the explicit formulation of $\gamma_{3}$ in (4.2).

TheOREM 4.4 (Piecewise $L^{\infty}\left(H^{-1}\right)$ and $L^{2}\left(H^{1}\right)$ error estimates). Assume $u$ is the solution of (1.1)-(1.4), $u_{h}^{n}$ is the numerical solution of scheme (3.9)-(3.10). Under the mesh constraints in Theorem 3.15 in [27], we have the following error estimate

$$
\begin{gathered}
\frac{1}{4}\left\|\nabla \widetilde{\Delta}_{h}^{-1} \theta^{\ell}\right\|_{0,2, h}^{2}+\frac{k^{2}}{4} \sum_{n=1}^{\ell}\left\|\nabla \widetilde{\Delta}_{h}^{-1} d_{t} \theta^{n}\right\|_{0,2, h}^{2}+\frac{\epsilon^{4} k}{16} \sum_{n=1}^{\ell}\left(\nabla \theta^{n}, \nabla \theta^{n}\right)_{h} \\
+\frac{k}{\epsilon} \sum_{n=1}^{\ell}\left\|\theta^{n}\right\|_{0,4, h}^{4} \leq C\left(\tilde{\rho}_{0}(\epsilon)|\ln h| h^{2}+\tilde{\rho}_{1}(\epsilon) k^{2}\right),
\end{gathered}
$$

where $\tilde{\rho}_{0}(\epsilon)$ and $\tilde{\rho}_{1}(\epsilon)$ are polynomial $\frac{1}{\epsilon}$-dependent functions and $\widetilde{\Delta}_{h}^{-1}$ is a discrete inverse Laplace operator defined in [27].

4.2. $L^{\infty}\left(L^{2}\right)$ and piecewise $L^{2}\left(H^{2}\right)$ error estimates. Based on Theorem 4.4, the $L^{\infty}\left(L^{2}\right)$ and piecewise $L^{2}\left(H^{2}\right)$ error estimates which depend on $\frac{1}{\epsilon}$ polynomially, instead of exponentially, are derived below. Notice that the Theorem 4.4 is used to circumvent the use of interpolation of $\|\cdot\|_{1,2, h}$ between $\|\cdot\|_{0,2, h}$ and $\|\cdot\|_{2,2, h}$, by which only the exponential dependence can be derived.

THEOREM 4.5. Assume $u$ is the solution of (1.1)-(1.4), $u_{h}^{n}$ is the numerical solution of scheme (3.9)-(3.10). Under the mesh constraints in Theorem 3.15 in [27] and (3.23), the following $L^{\infty}\left(L^{2}\right)$ and piecewise $L^{2}\left(H^{2}\right)$ error estimates hold

$$
\begin{aligned}
& \left\|\theta^{\ell}\right\|_{0,2, \Omega}^{2}+k \sum_{n=1}^{\ell}\left\|d_{t} \theta^{n}\right\|_{0,2, \Omega}^{2}+\epsilon k \sum_{n=1}^{\ell} a_{h}\left(\theta^{n}, \theta^{n}\right) \\
\leq & C \tilde{\rho}_{2}(\epsilon)|\ln h|^{2} h^{2}+C \tilde{\rho}_{3}(\epsilon)|\ln h| k^{2},
\end{aligned}
$$

where

$$
\begin{aligned}
& \tilde{\rho}_{2}(\epsilon):=\epsilon^{4} \rho_{3}(\epsilon)+\epsilon^{-2 \sigma_{1}-6} \rho_{4}(\epsilon)+\rho_{5}(\epsilon)+\epsilon^{-5} \tilde{\rho}_{0}(\epsilon)+\epsilon^{-2 \gamma_{1}-2 \gamma_{2}-2} \tilde{\rho}_{0}(\epsilon) \\
& \tilde{\rho}_{3}(\epsilon):=\rho_{3}(\epsilon)+\epsilon^{-5} \tilde{\rho}_{1}(\epsilon)+\epsilon^{-2 \gamma_{1}-2 \gamma_{2}-2} \tilde{\rho}_{1}(\epsilon)
\end{aligned}
$$

Proof. It follows from (3.9), (3.11), and (3.12) that for any $v_{h} \in S_{E}^{h}$,

$$
\begin{aligned}
& \left(d_{t} \theta^{n}, v_{h}\right)+\epsilon a_{h}\left(\theta^{n}, v_{h}\right) \\
= & {\left[\left(d_{t} P_{h} u, v_{h}\right)+\epsilon a_{h}\left(P_{h} u, v_{h}\right)\right]-\left[\left(d_{t} u_{h}^{n}, v_{h}\right)+\epsilon a_{h}\left(u_{h}^{n}, v_{h}\right)\right] } \\
= & -\left(d_{t} \rho^{n}, v_{h}\right)+\left(u_{t}+\epsilon \Delta^{2} u-\frac{1}{\epsilon} \Delta f(u)+\alpha u, v_{h}\right)+\left(R^{n}\left(u_{t t}\right), v_{h}\right) \\
& -\frac{1}{\epsilon}\left(f^{\prime}(u) \nabla P_{h} u, \nabla v_{h}\right)_{h}-\alpha\left(P_{h} u, v_{h}\right)+\frac{1}{\epsilon}\left(\nabla f\left(u_{h}^{n}\right), \nabla v_{h}\right)_{h} \\
= & \left(-d_{t} \rho^{n}+\alpha \rho^{n}, v_{h}\right)-\frac{1}{\epsilon}\left(f^{\prime}(u) \nabla P_{h} u-\nabla f\left(u_{h}^{n}\right), \nabla v_{h}\right)_{h} \\
& +\left(R^{n}\left(u_{t t}\right), v_{h}\right),
\end{aligned}
$$

where the remainder

$$
R^{n}\left(u_{t t}\right):=\frac{u\left(t_{n}\right)-u\left(t_{n-1}\right)}{k}-u_{t}\left(t_{n}\right)=-\frac{1}{k} \int_{t_{n-1}}^{t_{n}}\left(s-t_{n-1}\right) u_{t t}(s) \mathrm{d} s .
$$


Choosing $v_{h}=\theta^{n}$, taking summation over $n$ from 1 to $\ell$, multiplying $k$ on both sides of (4.6), we have

$$
\begin{aligned}
& \frac{1}{2}\left\|\theta^{\ell}\right\|_{0,2}^{2}+\frac{k}{2} \sum_{n=1}^{\ell}\left\|d_{t} \theta^{n}\right\|_{0,2}^{2}+\epsilon k \sum_{n=1}^{\ell} a_{h}\left(\theta^{n}, \theta^{n}\right) \\
= & k \sum_{n=1}^{\ell}\left(-d_{t} \rho^{n}+\alpha \rho^{n}, \theta^{n}\right)-\frac{k}{\epsilon} \sum_{n=1}^{\ell}\left(f^{\prime}(u) \nabla P_{h} u-\nabla f\left(u_{h}^{n}\right), \nabla \theta^{n}\right)_{h} \\
& +k \sum_{n=1}^{\ell}\left(R^{n}\left(u_{t t}\right), \theta^{n}\right):=I_{1}+I_{2}+I_{3} .
\end{aligned}
$$

Estimate of $I_{1}$ : The first term on the right hand side of (4.6) can be bounded by

$$
\begin{aligned}
I_{1} & =k \sum_{n=1}^{\ell}\left(-d_{t} \rho^{n}+\alpha \rho^{n}, \theta^{n}\right) \\
& \leq C k \sum_{n=1}^{\ell}\left\|d_{t} \rho^{n}\right\|_{0,2}^{2}+C k \sum_{n=1}^{\ell} \alpha^{2}\left\|\rho^{n}\right\|_{0,2}^{2}+C k \sum_{n=1}^{\ell}\left\|\theta^{n}\right\|_{0,2}^{2} \\
& \leq C\left(\epsilon^{4} \rho_{3}(\epsilon)+\epsilon^{-6} \rho_{4}(\epsilon)\right) h^{2}+C \rho_{5}(\epsilon)|\ln h| h^{2}+C k \sum_{n=1}^{\ell}\left\|\theta^{n}\right\|_{0,2}^{2},
\end{aligned}
$$

where by (3.20) and (3.21)

$$
\begin{aligned}
k \sum_{n=1}^{\ell}\left\|d_{t} \rho^{n}\right\|_{0,2}^{2} & =\frac{1}{k} \sum_{n=1}^{\ell}\left\|\int_{t_{n-1}}^{t_{n}} \rho_{t} \mathrm{~d} s\right\|_{0,2}^{2} \leq \sum_{n=1}^{\ell} \int_{t_{n-1}}^{t_{n}}\left\|\rho_{t}\right\|_{0,2}^{2} \mathrm{~d} s \\
& \leq \int_{0}^{T}\left\|\rho_{t}\right\|_{0,2}^{2} \mathrm{~d} s \leq C \epsilon^{4} \rho_{3}(\epsilon) h^{2}+C \rho_{5}(\epsilon)|\ln h| h^{2}, \\
k \sum_{n=1}^{\ell} \alpha^{2}\left\|\rho^{n}\right\|_{0,2}^{2} & \leq C \epsilon^{-6} \sup _{1 \leq n \leq \ell}\left\|\rho^{n}\right\|_{0,2}^{2} \leq C \epsilon^{-6} \rho_{4}(\epsilon) h^{2} .
\end{aligned}
$$

Estimate of $I_{2}$ : The second term on the right hand side of (4.8) can be written as

$$
\begin{aligned}
& -\frac{k}{\epsilon} \sum_{n=1}^{\ell}\left(f^{\prime}(u) \nabla P_{h} u-\nabla f\left(u_{h}^{n}\right), \nabla \theta^{n}\right)_{h} \\
= & -\frac{k}{\epsilon} \sum_{n=1}^{\ell}\left(f^{\prime}(u) \nabla P_{h} u-f^{\prime}\left(P_{h} u\right) \nabla P_{h} u, \nabla \theta^{n}\right)_{h} \\
& -\frac{k}{\epsilon} \sum_{n=1}^{\ell}\left(\nabla f\left(P_{h} u\right)-f^{\prime}\left(P_{h} u\right) \nabla u_{h}^{n}, \nabla \theta^{n}\right)_{h} \\
& -\frac{k}{\epsilon} \sum_{n=1}^{\ell}\left(f^{\prime}\left(P_{h} u\right) \nabla u_{h}^{n}-\nabla f\left(u_{h}^{n}\right), \nabla \theta^{n}\right)_{h}:=J_{1}+J_{2}+J_{3} .
\end{aligned}
$$

By (2.3), (3.20) and mesh condition (3.23), we have

$$
\left\|\nabla P_{h} u\right\|_{0,2}^{2} \leq\|\nabla u\|_{0,2}^{2}+C \leq \epsilon^{-2 \sigma_{1}-1} .
$$


Then, using (4.4) and the piecewise $L^{2}\left(H^{1}\right)$ error estimate given in Theorem 4.4, the first term on the right-hand side of (4.12) can be bounded below

$$
\begin{aligned}
J_{1} & =-\frac{3 k}{\epsilon} \sum_{n=1}^{\ell}\left(\rho^{n}\left(u+P_{h} u\right) \nabla P_{h} u, \nabla \theta^{n}\right)_{h} \\
& \leq \frac{C k}{\epsilon} \sum_{n=1}^{\ell}\left\|u+P_{h} u\right\|_{0, \infty}^{2}\left\|\rho^{n}\right\|_{0, \infty}^{2}\left\|\nabla P_{h} u\right\|_{0,2}^{2}+\frac{C k}{\epsilon} \sum_{n=1}^{\ell}\left(\nabla \theta^{n}, \nabla \theta^{n}\right)_{h} \\
& \leq C \epsilon^{-2 \sigma_{1}-6} \rho_{4}(\epsilon) h^{2}+C \epsilon^{-5} \tilde{\rho}_{0}(\epsilon)|\ln h| h^{2}+C \epsilon^{-5} \tilde{\rho}_{1}(\epsilon) k^{2} .
\end{aligned}
$$

Again, thanks to the piecewise $L^{2}\left(H^{1}\right)$ error estimate given in Theorem 4.4, the second term on the right-hand side of (4.12) can be written as

$$
\begin{aligned}
J_{2} & =-\frac{k}{\epsilon} \sum_{n=1}^{\ell}\left(f^{\prime}\left(P_{h} u\right) \nabla \theta^{n}, \nabla \theta^{n}\right)_{h} \leq \frac{C k}{\epsilon} \sum_{n=1}^{\ell}\left(\nabla \theta^{n}, \nabla \theta^{n}\right)_{h} \\
& \leq C \epsilon^{-5} \tilde{\rho}_{0}(\epsilon)|\ln h| h^{2}+C \epsilon^{-5} \tilde{\rho}_{1}(\epsilon) k^{2} .
\end{aligned}
$$

By the discrete Sobolev inequality and Theorem 3.14 in [27], we have for any $n$,

$$
\left\|u_{h}^{n}\right\|_{1, \infty, h} \leq C|\ln h|^{\frac{1}{2}}\left\|u_{h}^{n}\right\|_{2,2, h} \leq C \epsilon^{-\gamma_{2}}|\ln h|^{\frac{1}{2}} .
$$

Then, the third term on the right-hand side of (4.12) can be bounded by

$$
\begin{aligned}
J_{3} & =-\frac{3 k}{\epsilon} \sum_{n=1}^{\ell}\left(\theta^{n}\left(P_{h} u+u_{h}\right) \nabla u_{h}^{n}, \nabla \theta^{n}\right) \\
& \leq C k \sum_{n=1}^{\ell}\left\|\theta^{n}\right\|_{0,2}^{2}+\frac{C k}{\epsilon^{2}} \sum_{n=1}^{\ell}\left\|P_{h} u+u_{h}^{n}\right\|_{0, \infty}^{2}\left\|u_{h}^{n}\right\|_{1, \infty, h}^{2}\left\|\nabla \theta^{n}\right\|_{0,2}^{2} \\
& \leq C k \sum_{n=1}^{\ell}\left\|\theta^{n}\right\|_{0,2}^{2}+C \epsilon^{-2 \gamma_{1}-2 \gamma_{2}-2}|\ln h| k \sum_{n=1}^{\ell}\left\|\nabla \theta^{n}\right\|_{0,2}^{2} \\
& \leq C k \sum_{n=1}^{\ell}\left\|\theta^{n}\right\|_{0,2}^{2}+C \epsilon^{-2 \gamma_{1}-2 \gamma_{2}-2}\left(\tilde{\rho}_{0}(\epsilon)|\ln h|^{2} h^{2}+\tilde{\rho}_{1}(\epsilon)|\ln h| k^{2}\right) .
\end{aligned}
$$

Estimate of $I_{3}$ : The third term on the right hand side of (4.6) can be bounded by

$$
\begin{aligned}
I_{3}=k \sum_{n=1}^{\ell}\left(R^{n}\left(u_{t t}\right), \theta^{n}\right) & \leq C k \sum_{n=1}^{\ell}\left\|R^{n}\left(u_{t t}\right)\right\|_{0,2}^{2}+C k \sum_{n=1}^{\ell}\left\|\theta^{n}\right\|_{0,2}^{2} \\
& \leq C \rho_{3}(\epsilon) k^{2}+C k \sum_{n=1}^{\ell}\left\|\theta^{n}\right\|_{0,2}^{2}
\end{aligned}
$$

where by (2.13) and (4.7),

$$
\begin{aligned}
k \sum_{n=1}^{\ell}\left\|R^{n}\left(u_{t t}\right)\right\|_{0,2}^{2} & \leq \frac{1}{k} \sum_{n=1}^{\ell}\left(\int_{t_{n-1}}^{t_{n}}\left(s-t_{n-1}\right)^{2} \mathrm{~d} s\right)\left(\int_{t_{n-1}}^{t_{n}}\left\|u_{t t}(s)\right\|_{0,2}^{2} \mathrm{~d} s\right) \\
& \leq C \rho_{3}(\epsilon) k^{2} .
\end{aligned}
$$


$L^{\infty}\left(L^{2}\right)$ and piecewise $L^{2}\left(H^{2}\right)$ error estimates: Taking (4.9), (4.13), (4.14), (4.16), (4.16) into (4.8), we have

$$
\begin{aligned}
& \frac{1}{2}\left\|\theta^{\ell}\right\|_{0,2}^{2}+\frac{k}{2} \sum_{n=1}^{\ell}\left\|d_{t} \theta^{n}\right\|_{0,2}^{2}+\epsilon k \sum_{n=1}^{\ell} a_{h}\left(\theta^{n}, \theta^{n}\right) \\
\leq & C k \sum_{n=1}^{\ell}\left\|\theta^{n}\right\|_{0,2}^{2} \\
& +C\left(\epsilon^{4} \rho_{3}(\epsilon)+\epsilon^{-2 \sigma_{1}-6} \rho_{4}(\epsilon)\right) h^{2} \\
& +C\left(\rho_{5}(\epsilon)+\epsilon^{-5} \tilde{\rho}_{0}(\epsilon)\right)|\ln h| h^{2}+\epsilon^{-2 \gamma_{1}-2 \gamma_{2}-2} \tilde{\rho}_{0}(\epsilon)|\ln h|^{2} h^{2} \\
& +C\left(\rho_{3}(\epsilon)+\epsilon^{-5} \tilde{\rho}_{1}(\epsilon)\right) k^{2}+C \epsilon^{-2 \gamma_{1}-2 \gamma_{2}-2} \tilde{\rho}_{1}(\epsilon)|\ln h| k^{2} .
\end{aligned}
$$

The desired result (4.5) is therefore obtained by the Gronwall's inequality.

4.3. Piecewise $L^{\infty}\left(H^{2}\right)$ and $L^{\infty}\left(L^{\infty}\right)$ error estimates. In this subsection, we give the $\left\|\theta^{\ell}\right\|_{2,2, h}^{2}$ estimate by taking the summation by parts in time and integration by parts in space, and using the special properties of the Morley element. The $\left\|\theta^{\ell}\right\|_{2,2, h}^{2}$ estimate below is "almost" optimal with respect to time and space.

TheOrem 4.6. Assume $u$ is the solution of (1.1)-(1.4), $u_{h}^{n}$ is the numerical solution of scheme (3.9)-(3.10). Under the mesh constraints in Theorem 3.15 in [27] and (3.23), the following piecewise $L^{\infty}\left(H^{2}\right)$ error estimate holds

$$
\begin{aligned}
& k \sum_{n=1}^{\ell}\left\|d_{t} \theta^{n}\right\|_{L^{2}}^{2}+\epsilon k^{2} \sum_{n=1}^{\ell} a_{h}\left(d_{t} \theta^{n}, d_{t} \theta^{n}\right)+\epsilon\left\|\theta^{\ell}\right\|_{2,2, h}^{2} \\
\leq & C \tilde{\rho}_{4}(\epsilon)|\ln h|^{2} h^{2}+C \tilde{\rho}_{5}(\epsilon)|\ln h| k^{2},
\end{aligned}
$$

where

$$
\begin{aligned}
\tilde{\rho}_{4}(\epsilon)= & \epsilon^{-2 \sigma_{1}-1} \rho_{3}(\epsilon)+\epsilon^{-4} \rho_{0}(\epsilon) \rho_{4}(\epsilon)+\epsilon^{-2 \sigma_{1}-5} \rho_{5}(\epsilon) \\
+ & \left(\epsilon^{-4 \gamma_{1}-3}+\epsilon^{-4 \gamma_{2}-2}+\epsilon^{-\max \left\{2 \sigma_{1}+5,2 \sigma_{3}+2\right\}-2}\right. \\
& \left.\quad+\epsilon^{2 \gamma_{1}-\max \left\{2 \sigma_{1}+\frac{13}{2}, 2 \sigma_{3}+\frac{7}{2}, 2 \sigma_{2}+4,2 \sigma_{4}\right\}-1}\right) \tilde{\rho}_{2}(\epsilon), \\
\tilde{\rho}_{5}(\epsilon)=( & \epsilon^{-4 \gamma_{1}-3}+\epsilon^{-4 \gamma_{2}-2}+\epsilon^{-\max \left\{2 \sigma_{1}+5,2 \sigma_{3}+2\right\}-2} \\
& \left.+\epsilon^{2 \gamma_{1}-\max \left\{2 \sigma_{1}+\frac{13}{2}, 2 \sigma_{3}+\frac{7}{2}, 2 \sigma_{2}+4,2 \sigma_{4}\right\}-1}\right) \tilde{\rho}_{3}(\epsilon) .
\end{aligned}
$$

Proof. Choosing $v_{h}=\theta^{n}-\theta^{n-1}=k d_{t} \theta^{n}$ in (4.6), taking summation over $n$ from 1 to $\ell$, we get

$$
\begin{aligned}
& k \sum_{n=1}^{\ell}\left\|d_{t} \theta^{n}\right\|_{L^{2}}^{2}+\frac{\epsilon}{2} a_{h}\left(\theta^{\ell}, \theta^{\ell}\right)+\frac{\epsilon k^{2}}{2} \sum_{n=1}^{\ell} a_{h}\left(d_{t} \theta^{n}, d_{t} \theta^{n}\right) \\
= & k \sum_{n=1}^{\ell}\left(-d_{t} \rho^{n}+\alpha \rho^{n}, d_{t} \theta^{n}\right)-\frac{k}{\epsilon} \sum_{n=1}^{\ell}\left(f^{\prime}(u) \nabla P_{h} u-\nabla f\left(u_{h}^{n}\right), \nabla\left(d_{t} \theta^{n}\right)\right)_{h} \\
& +k \sum_{n=1}^{\ell}\left(R^{n}\left(u_{t t}\right), d_{t} \theta^{n}\right):=I_{1}+I_{2}+I_{3} .
\end{aligned}
$$


Here we use the fact that

$$
\epsilon a_{h}\left(\theta^{n}, \theta^{n}-\theta^{n-1}\right)=\frac{\epsilon k^{2}}{2} a_{h}\left(d_{t} \theta^{n}, d_{t} \theta^{n}\right)+\frac{\epsilon}{2} a_{h}\left(\theta^{n}, \theta^{n}\right)-\frac{\epsilon}{2} a_{h}\left(\theta^{n-1}, \theta^{n-1}\right) .
$$

Estimates of $I_{1}$ and $I_{3}$ : Similar to (4.9), using (4.10) and (4.11), we have

$$
\begin{aligned}
I_{1} & \leq C k \sum_{n=1}^{\ell}\left\|d_{t} \rho^{n}\right\|_{L^{2}}^{2}+C k \sum_{n=1}^{\ell} \alpha^{2}\left\|\rho^{n}\right\|_{L^{2}}^{2}+\frac{k}{8} \sum_{n=1}^{\ell}\left\|d_{t} \theta^{n}\right\|_{L^{2}}^{2} \\
& \leq C\left(\epsilon^{4} \rho_{3}(\epsilon)+\epsilon^{-6} \rho_{4}(\epsilon)\right) h^{2}+C \rho_{5}(\epsilon)|\ln h| h^{2}+\frac{k}{8} \sum_{n=1}^{\ell}\left\|d_{t} \theta^{n}\right\|_{0,2, h}^{2} .
\end{aligned}
$$

From (4.17) and (4.18), we also obtain the estimate of $I_{3}$ below

$$
\begin{aligned}
I_{3}=k \sum_{n=1}^{\ell}\left(R^{n}\left(u_{t t}\right), d_{t} \theta^{n}\right) & \leq C k \sum_{n=1}^{\ell}\left\|R^{n}\left(u_{t t}\right)\right\|_{L^{2}}^{2}+\frac{k}{8} \sum_{n=1}^{\ell}\left\|d_{t} \theta^{n}\right\|_{0,2}^{2} \\
& \leq C \rho_{3}(\epsilon) k^{2}+\frac{k}{8} \sum_{n=1}^{\ell}\left\|d_{t} \theta^{n}\right\|_{0,2}^{2} .
\end{aligned}
$$

Estimate of $I_{2}$ : Next we bound the more complicated term $I_{2}$. Using integration by parts, we have

$$
\begin{aligned}
I_{2}= & -\frac{k}{\epsilon} \sum_{n=1}^{\ell}\left(f^{\prime}(u) \nabla P_{h} u-\nabla f\left(P_{h} u\right), d_{t} \nabla \theta^{n}\right)_{h}-\frac{k}{\epsilon} \sum_{n=1}^{\ell}\left(\nabla\left(f\left(P_{h} u\right)-f\left(u_{h}^{n}\right)\right), d_{t} \nabla \theta^{n}\right)_{h} \\
= & -\frac{k}{\epsilon} \sum_{n=1}^{\ell}\left(f^{\prime}(u) \nabla P_{h} u-\nabla f\left(P_{h} u\right), d_{t} \nabla \theta^{n}\right)_{h}+\frac{k}{\epsilon} \sum_{n=1}^{\ell}\left(f\left(P_{h} u\right)-f\left(u_{h}^{n}\right), d_{t} \Delta \theta^{n}\right)_{h} \\
& -\frac{k}{\epsilon} \sum_{n=1}^{\ell} \sum_{E \in \mathcal{E}_{h}}\left(\left\{f\left(P_{h} u\right)-f\left(u_{h}^{n}\right)\right\}, d_{t} \llbracket \nabla \theta^{n} \rrbracket\right)_{E} \\
& -\frac{k}{\epsilon} \sum_{n=1}^{\ell} \sum_{E \in \mathcal{E}_{h}}\left(\llbracket f\left(P_{h} u\right)-f\left(u_{h}^{n}\right) \rrbracket,\left\{\nabla d_{t} \theta^{n}\right\}\right)_{E}:=J_{1}+J_{2}+J_{3}+J_{4} .
\end{aligned}
$$

Here we adopt the standard DG notation and the DG identity, see [5, Equ. (3.3)]. Next we bound $J_{1}$ to $J_{4}$ respectively.

- Estimate of $J_{1}$. Using summation by parts in Lemma 4.1, we have

$$
J_{1}=\frac{k}{\epsilon} \sum_{n=1}^{\ell}\left(d_{t}\left(\rho\left(u+P_{h} u\right) \nabla P_{h} u\right), \nabla \theta^{n-1}\right)_{h}-\frac{1}{\epsilon}\left(\rho^{\ell}\left(u^{\ell}+P_{h} u^{\ell}\right) \nabla P_{h} u^{\ell}, \nabla \theta^{\ell}\right)_{h}
$$


Thanks to (2.3), (2.7), (2.9), (3.20), (3.21), (3.24), and the piecewise $L^{2}\left(H^{1}\right)$ estimate in Theorem 4.4, the first term on the right hand side of (4.25) can be bounded by

$$
\begin{aligned}
& \frac{k}{\epsilon} \sum_{n=1}^{\ell}\left(d_{t}\left(\rho\left(u+P_{h} u\right) \nabla P_{h} u\right), \nabla \theta^{n-1}\right)_{h} \\
\leq & \frac{1}{k} \sum_{n=1}^{\ell}\left\|\int_{t_{n-1}}^{t_{n}}\left(\rho\left(u+P_{h} u\right) \nabla P_{h} u\right)_{t} \mathrm{~d} s\right\|_{0,2}^{2}+C \epsilon^{-2} k \sum_{n=1}^{\ell}\left|\theta^{n-1}\right|_{1,2, h}^{2} \\
\leq & \operatorname{ess~sup}_{t \in[0, T]}\left\|\nabla P_{h} u\right\|_{0,2}^{2} \int_{0}^{T}\left\|\rho_{t}\right\|_{0, \infty}^{2} \mathrm{~d} s+\operatorname{ess~sup}_{t \in[0, T]}\|\rho\|_{0, \infty}^{2} \int_{0}^{T}\left\|\nabla\left(P_{h} u\right)_{t}\right\|_{0,2}^{2} \mathrm{~d} s \\
+ & \operatorname{ess} \sup _{t \in[0, T]}\|\rho\|_{0, \infty}^{2}\left\|\nabla P_{h} u\right\|_{0,2}^{2} \int_{0}^{T}\left\|u_{t}+\left(P_{h} u\right)_{t}\right\|_{0, \infty}^{2} \mathrm{~d} s+C \epsilon^{-2} k \sum_{n=1}^{\ell}\left|\theta^{n-1}\right|_{1,2, h}^{2} \\
\leq & C \epsilon^{-2 \sigma_{1}-1}\left(\rho_{3}(\epsilon)+\epsilon^{-4} \rho_{5}(\epsilon)|\ln h|\right) h^{2}+C \epsilon^{-4} \rho_{0}(\epsilon) \rho_{4}(\epsilon) h^{2} \\
& +C \epsilon^{-2 \sigma_{1}-6-\max \left\{2 \sigma_{1}+\frac{13}{2}, 2 \sigma_{3}+\frac{7}{2}, 2 \sigma_{2}+4,2 \sigma_{4}\right\}} \rho_{4}(\epsilon) h^{2} \\
& +C \epsilon^{-6} \tilde{\rho}_{0}(\epsilon)|\ln h| h^{2}+C \epsilon^{-6} \tilde{\rho}_{1}(\epsilon) k^{2} .
\end{aligned}
$$

Thanks to (2.3), (3.20) and the $L^{\infty}\left(L^{2}\right)$ estimate in Theorem 4.5, the second term on the right hand of (4.25) can be bounded by

$$
\begin{aligned}
& -\frac{1}{\epsilon}\left(\rho^{\ell}\left(u^{\ell}+P_{h} u^{\ell}\right) \nabla P_{h} u^{\ell}, \nabla \theta^{\ell}\right)_{h} \\
\leq & C \epsilon^{-2}\left\|\rho^{l}\right\|_{0, \infty}^{2}\left|P_{h} u^{l}\right|_{1,2, h}^{2}+C \epsilon^{-1}\|\theta\|_{0,2}^{2}+\frac{\epsilon}{8} a_{h}\left(\theta^{l}, \theta^{l}\right) \\
\leq & C \epsilon^{-2 \sigma_{1}-7} \rho_{4}(\epsilon) h^{2}+C \epsilon^{-1} \tilde{\rho}_{2}(\epsilon)|\ln h|^{2} h^{2}+C \epsilon^{-1} \tilde{\rho}_{3}(\epsilon)|\ln h| k^{2}+\frac{\epsilon}{8} a_{h}\left(\theta^{l}, \theta^{l}\right) .
\end{aligned}
$$

Combining (4.26) and (4.27), simplifying the coefficients according to the definition of $\rho_{i}(\epsilon)$ and $\tilde{\rho}_{i}(\epsilon)$, we obtain the bound for $J_{1}$ :

$$
\begin{aligned}
J_{1} \leq & C\left(\epsilon^{-2 \sigma_{1}-1} \rho_{3}(\epsilon)+\epsilon^{-4} \rho_{0}(\epsilon) \rho_{4}(\epsilon)+\epsilon^{-2 \sigma_{1}-5} \rho_{5}(\epsilon)+\epsilon^{-1} \tilde{\rho}_{2}(\epsilon)\right)|\ln h|^{2} h^{2} \\
& +C \epsilon^{-1} \tilde{\rho}_{3}(\epsilon)|\ln h| k^{2}+\frac{\epsilon}{8} a_{h}\left(\theta^{l}, \theta^{l}\right)
\end{aligned}
$$

- Estimate of $J_{2}$. Define $f\left(P_{h} u\right)-f\left(u_{h}^{n}\right):=M^{n} \theta^{n}$, where $M^{n}$ is given as

$$
M^{n}:=\left(P_{h} u\left(t_{n}\right)\right)^{2}+P_{h} u\left(t_{n}\right) u_{h}^{n}+\left(u_{h}^{n}\right)^{2}-1 .
$$

Using summation by parts in Lemma 4.1, we have

$$
\begin{aligned}
J_{2} & =-\frac{k}{\epsilon} \sum_{n=1}^{\ell}\left(d_{t}\left(M^{n} \theta^{n}\right), \Delta \theta^{n-1}\right)_{h}+\frac{1}{\epsilon}\left(M^{l} \theta^{l}, \Delta \theta^{l}\right)_{h} \\
& \leq \frac{C k}{\epsilon} \sum_{n=1}^{\ell}\left\|d_{t}\left(M^{n} \theta^{n}\right)\right\|_{0,2}|\theta|_{2,2, h}+\frac{C}{\epsilon}\left\|M^{l} \theta^{l}\right\|_{0,2}\left|\theta^{l}\right|_{2,2, h} .
\end{aligned}
$$


Since $d_{t} u_{h}^{n}=d_{t}\left(P_{h} u^{n}\right)-d_{t} \theta^{n}$, a direct calculation shows that

$$
\begin{aligned}
d_{t}\left(M^{n} \theta^{n}\right)= & \theta^{n} d_{t} M^{n}+M^{n-1} d_{t} \theta^{n} \\
= & M^{n-1} d_{t} \theta^{n}+\theta^{n}\left(P_{h} u^{n}+P_{h} u^{n-1}\right) d_{t}\left(P_{h} u^{n}\right) \\
& +\theta^{n} u_{h}^{n} d_{t}\left(P_{h} u^{n}\right)+\theta^{n} P_{h} u^{n-1} d_{t}\left(P_{h} u^{n}\right)-\theta^{n} P_{h} u^{n-1} d_{t} \theta^{n} \\
& +\theta^{n}\left(u_{h}^{n}+u_{h}^{n-1}\right) d_{t}\left(P_{h} u^{n}\right)-\theta^{n}\left(u_{h}^{n}+u_{h}^{n-1}\right) d_{t} \theta^{n} \\
= & \left(M^{n-1}-\theta^{n} P_{h} u^{n-1}-\theta^{n}\left(u_{h}^{n}+u_{h}^{n-1}\right)\right) d_{t} \theta^{n} \\
& +\left(P_{h} u^{n}+2 P_{h} u^{n-1}+2 u_{h}^{n}+u_{h}^{n-1}\right) \theta^{n} d_{t}\left(P_{h} u^{n}\right) .
\end{aligned}
$$

Using the $L^{2}\left(H^{2}\right)$ error estimate (4.5) and the assumption on the $L^{\infty}$ bound of $u_{h}^{n}$, we get

$$
\begin{aligned}
& \frac{C k}{\epsilon} \sum_{n=1}^{\ell}\left\|d_{t}\left(M^{n} \theta^{n}\right)\right\|_{0,2}\left|\theta^{n}\right|_{2,2, h} \\
\leq & C \epsilon^{-2 \gamma_{1}-1} k \sum_{n=1}^{\ell}\left\|d_{t} \theta^{n}\right\|_{0,2}\left|\theta^{n}\right|_{2,2, h}+C \epsilon^{-\gamma_{1}-1} k \sum_{n=1}^{\ell}\left\|\theta^{n} d_{t}\left(P_{h} u\right)\right\|_{0,2}\left|\theta^{n}\right|_{2,2, h} \\
\leq & \frac{k}{8} \sum_{n=1}^{\ell}\left\|d_{t} \theta^{n}\right\|_{0,2}^{2}+C \epsilon^{-4 \gamma_{1}-2} k \sum_{n=1}^{\ell}|\theta|_{2,2, h}^{2}+C \epsilon^{2 \gamma_{1}} k \sum_{n=1}^{\ell}\left\|\theta d_{t}\left(P_{h} u\right)\right\|_{0,2}^{2} \\
\leq & \frac{k}{8} \sum_{n=1}^{\ell}\left\|d_{t} \theta^{n}\right\|_{0,2}^{2}+C \epsilon^{-4 \gamma_{1}-3}\left(\tilde{\rho}_{2}(\epsilon)|\ln h|^{2} h^{2}+\tilde{\rho}_{3}(\epsilon)|\ln h| k^{2}\right) \\
& +C \epsilon^{2 \gamma_{1}-\max \left\{2 \sigma_{1}+\frac{13}{2}, 2 \sigma_{3}+\frac{7}{2}, 2 \sigma 2+4,2 \sigma_{4}\right\}-1}\left(\tilde{\rho}_{2}(\epsilon)|\ln h|^{2} h^{2}+\tilde{\rho}_{3}(\epsilon)|\ln h| k^{2}\right),
\end{aligned}
$$

where by (2.7) and the $L^{\infty}\left(L^{2}\right)$ error estimate (4.5),

$$
\begin{aligned}
& k \sum_{n=1}^{\ell}\left\|\theta d_{t}\left(P_{h} u\right)\right\|_{0,2}^{2} \\
\leq & \sup _{1 \leq n \leq \ell}\left\|\theta^{n}\right\|_{0,2}^{2} \frac{1}{k}\left\|\int_{t_{n-1}}^{t_{n}}\left(P_{h} u\right)_{t} \mathrm{~d} s\right\|_{0, \infty}^{2} \\
\leq & \sup _{1 \leq n \leq \ell}\left\|\theta^{n}\right\|_{0,2}^{2} \int_{0}^{T}\left\|\left(P_{h} u\right)_{t}\right\|_{0, \infty}^{2} \mathrm{~d} s \\
\leq & C \epsilon^{-\max \left\{2 \sigma_{1}+\frac{13}{2}, 2 \sigma_{3}+\frac{7}{2}, 2 \sigma 2+4,2 \sigma_{4}\right\}-1}\left(\tilde{\rho}_{2}(\epsilon)|\ln h|^{2} h^{2}+\tilde{\rho}_{3}(\epsilon)|\ln h| k^{2}\right) .
\end{aligned}
$$

And the second term on the right hand side of (4.29) can be bounded by

$$
\begin{aligned}
\frac{C}{\epsilon}\left\|M^{l} \theta^{l}\right\|_{0,2}\left|\theta^{l}\right|_{2,2, h} & \leq C^{-4 \gamma_{1}-3}\left\|\theta^{l}\right\|_{0,2}^{2}+\frac{\epsilon}{8} a_{h}\left(\theta^{l}, \theta^{l}\right) \\
& \leq C \epsilon^{-4 \gamma_{1}-3}\left(\tilde{\rho}_{2}(\epsilon)|\ln h|^{2} h^{2}+\tilde{\rho}_{3}(\epsilon)|\ln h| k^{2}\right)+\frac{\epsilon}{8} a_{h}\left(\theta^{l}, \theta^{l}\right) .
\end{aligned}
$$

Combining (4.30) and (4.31), we obtain the bound for $J_{2}$ :

$$
\begin{aligned}
J_{2} \leq & \frac{k}{8} \sum_{n=1}^{\ell}\left\|d_{t} \theta^{n}\right\|_{0,2}^{2}+\frac{\epsilon}{8} a_{h}\left(\theta^{l}, \theta^{l}\right)+C \epsilon^{-4 \gamma_{1}-3}\left(\tilde{\rho}_{2}(\epsilon)|\ln h|^{2} h^{2}+\tilde{\rho}_{3}(\epsilon)|\ln h| k^{2}\right) \\
& +C \epsilon^{2 \gamma_{1}-\max \left\{2 \sigma_{1}+\frac{13}{2}, 2 \sigma_{3}+\frac{7}{2}, 2 \sigma_{2}+4,2 \sigma_{4}\right\}-1}\left(\tilde{\rho}_{2}(\epsilon)|\ln h|^{2} h^{2}+\tilde{\rho}_{3}(\epsilon)|\ln h| k^{2}\right) .
\end{aligned}
$$


- Estimate of $J_{3}$. Notice that $\theta^{n} \in S_{E}^{h}$ and

$$
\int_{E} \llbracket \nabla \theta^{n} \rrbracket \mathrm{d} S=0 \quad \forall E \in \mathcal{E}_{h}
$$

Using summation by parts in Lemma 4.1, Lemma 2.2 in [14] and inverse inequality, we have

$$
\begin{aligned}
J_{3} & =\frac{k}{\epsilon} \sum_{n=1}^{\ell} \sum_{E \in \mathcal{E}_{h}}\left(d_{t}\left\{M^{n} \theta^{n}\right\}, \llbracket \nabla \theta^{n-1} \rrbracket\right)_{E}-\frac{1}{\epsilon} \sum_{E \in \mathcal{E}_{h}}\left(\left\{M^{\ell} \theta^{\ell}\right\}, \llbracket \nabla \theta^{\ell} \rrbracket\right)_{E} \\
& \leq \frac{C k}{\epsilon} \sum_{n=1}^{\ell}\left\|d_{t}\left(M^{n} \theta^{n}\right)\right\|_{0,2}|\theta|_{2,2, h}+\frac{C}{\epsilon}\left\|M^{\ell} \theta^{\ell}\right\|_{0,2}\left|\theta^{l}\right|_{2,2, h} .
\end{aligned}
$$

Hence, $J_{3}$ has the same bound as $J_{2}$.

- Estimate of $J_{4}$. Since $P_{h} u$ and $u_{h}$ are continuous at vertexes of $\mathcal{T}_{h}$, thanks to Lemma 2.6 in [14], we have

$$
\begin{aligned}
J_{4} & \leq \frac{C k}{\epsilon} \sum_{n=1}^{\ell} h\left|M^{n} \theta^{n}\right|_{2,2, h}\left|d_{t} \theta^{n}\right|_{1,2, h} \\
& \leq \frac{C k}{\epsilon} \sum_{n=1}^{\ell}\left|M^{n} \theta^{n}\right|_{2,2, h}\left\|d_{t} \theta^{n}\right\|_{0,2} \\
& \leq \frac{C k}{\epsilon^{2}} \sum_{n=1}^{\ell}\left|M^{n} \theta^{n}\right|_{2,2, h}^{2}+\frac{k}{8} \sum_{n=1}^{\ell}\left\|d_{t} \theta^{n}\right\|_{0,2}^{2} .
\end{aligned}
$$

Using the piecewise $L^{2}\left(H^{2}\right)$ estimate given in Theorem 4.4, we have

$$
\begin{aligned}
& \frac{C k}{\epsilon^{2}} \sum_{n=1}^{\ell}\left|M^{n} \theta^{n}\right|_{2,2, h}^{2} \\
\leq & \frac{C k}{\epsilon^{2}} \sum_{n=1}^{\ell}\left(\left\|M^{n}\right\|_{0, \infty}^{2}\left|\theta^{n}\right|_{2,2, h}^{2}+\left|M^{n}\right|_{1,4, h}^{2}\left|\theta^{n}\right|_{1,4, h}^{2}+\left|M^{n}\right|_{2,2, h}^{2}\left\|\theta^{n}\right\|_{0, \infty}^{2}\right) \\
\leq & \frac{C}{\epsilon^{2}} \sup _{1 \leq n \leq \ell}\left\|M^{n}\right\|_{2,2, h}^{2} k \sum_{n=1}^{\ell}\left\|\theta^{n}\right\|_{2,2, h}^{2} \\
\leq & C\left(\epsilon^{-4 \gamma_{2}-2}+\epsilon^{-\max \left\{2 \sigma_{1}+5,2 \sigma_{3}+2\right\}-2}\right)\left(\tilde{\rho}_{2}(\epsilon)|\ln h|^{2} h^{2}+\tilde{\rho}_{3}(\epsilon)|\ln h| k^{2}\right),
\end{aligned}
$$

where by (2.6) and the fact that $\left\|u_{h}^{n}\right\|_{2,2, h} \leq C \epsilon^{-\gamma_{2}}$ (c.f. [27, Theorem 3.14])

$$
\begin{aligned}
\left\|M^{n}\right\|_{2,2, h} \leq & C\left(\left\|\left(P_{h} u^{n}\right)^{2}\right\|_{2,2, h}+\left\|u_{h}^{n} P_{h} u^{n}\right\|_{2,2, h}+\left\|\left(u_{h}^{n}\right)^{2}\right\|_{2,2, h}\right) \\
\leq & C\left(\left\|P_{h} u^{n}\right\|_{2,2, h}+\left\|P_{h} u^{n}\right\|_{1,4, h}^{2}+\left\|u_{h}\right\|_{0, \infty}\left\|u_{h}^{n}\right\|_{2,2, h}+\left\|u_{h}^{n}\right\|_{1,4, h}^{2}\right. \\
& \left.+\left\|u_{h}^{n}\right\|_{2,2, h}+\left\|u_{h}^{n}\right\|_{0, \infty}\left\|P_{h} u^{n}\right\|_{2,2, h}+\left\|u_{h}^{n}\right\|_{1,4, h}\left\|P_{h} u^{n}\right\|_{1,4, h}\right) \\
\leq & C\left(\epsilon^{-2 \gamma_{2}}+\epsilon^{-\max \left\{2 \sigma_{1}+5,2 \sigma_{3}+2\right\}}\right) .
\end{aligned}
$$

Piecewise $L^{\infty}\left(H^{2}\right)$ error estimate: Taking (4.22), (4.23), (4.28), (4.32) and (4.33) 
into (4.21), we obtain

$$
\begin{aligned}
& \frac{k}{8} \sum_{n=1}^{\ell}\left\|d_{t} \theta^{n}\right\|_{L^{2}}^{2}+\frac{\epsilon}{8} a_{h}\left(\theta^{\ell}, \theta^{\ell}\right)+\frac{\epsilon k^{2}}{2} \sum_{n=1}^{\ell} a_{h}\left(d_{t} \theta^{n}, d_{t} \theta^{n}\right) \\
\leq & C\left(\epsilon^{4} \rho_{3}(\epsilon)+\epsilon^{-6} \rho_{4}(\epsilon)\right) h^{2}+C \rho_{5}(\epsilon)|\ln h|^{2} h^{2}+C \rho_{3}(\epsilon) k^{2} \\
& +C\left(\epsilon^{-2 \sigma_{1}-1} \rho_{3}(\epsilon)+\epsilon^{-4} \rho_{0}(\epsilon) \rho_{4}(\epsilon)+\epsilon^{-2 \sigma_{1}-5} \rho_{5}(\epsilon)+\epsilon^{-1} \tilde{\rho}_{2}(\epsilon)\right)|\ln h|^{2} h^{2} \\
& +C \epsilon^{-1} \tilde{\rho}_{3}(\epsilon)|\ln h| k^{2}+C \epsilon^{-4 \gamma_{1}-3}\left(\tilde{\rho}_{2}(\epsilon)|\ln h|^{2} h^{2}+\tilde{\rho}_{3}(\epsilon)|\ln h| k^{2}\right) \\
& +C \epsilon^{-\max \left\{2 \sigma_{1}+\frac{13}{2}, 2 \sigma_{3}+\frac{7}{2}, 2 \sigma_{2}+4,2 \sigma_{4}\right\}-1}\left(\tilde{\rho}_{2}(\epsilon)|\ln h|^{2} h^{2}+\tilde{\rho}_{3}(\epsilon)|\ln h| k^{2}\right) \\
& +C\left(\epsilon^{-4 \gamma_{2}-2}+\epsilon^{-\max \left\{2 \sigma_{1}+5,2 \sigma_{3}+2\right\}-2}\right)\left(\tilde{\rho}_{2}(\epsilon)|\ln h|^{2} h^{2}+\tilde{\rho}_{3}(\epsilon)|\ln h| k^{2}\right) .
\end{aligned}
$$

Then the theorem can be proved by simplifying the coefficients according to the definitions of $\rho_{i}(\epsilon)$ and $\tilde{\rho}_{i}(\epsilon)$.

REMARK 2. If the summation by part for time and integration by part for space techniques are not employed simultaneously, one can only obtain a coarse estimate

$$
\begin{aligned}
& \left\|\theta^{\ell}\right\|_{2,2, h}^{2}+k \sum_{n=1}^{\ell}\left\|d_{t} \theta^{n}\right\|_{L^{2}}^{2}+\epsilon k^{2} \sum_{n=1}^{\ell} a_{h}\left(d_{t} \theta^{n}, d_{t} \theta^{n}\right) \\
\leq & C k^{-\frac{1}{2}}\left(\epsilon^{-\gamma_{4}}|\ln h|^{2} h^{2}+\epsilon^{-\gamma_{5}}|\ln h| k\right),
\end{aligned}
$$

where $\gamma_{4}, \gamma_{5}$ denote some positive constants.

Finally, using (4.4), Theorem 4.6 and the Sobolev embedding theorem, we can prove the desired $L^{\infty}\left(L^{\infty}\right)$ error estimate.

THEOREM 4.7. Assume $u$ is the solution of (1.1)-(1.4), $u_{h}^{n}$ is the numerical solution of scheme (3.9)-(3.10). Under the mesh constraints in Theorem 3.15 in [27] and (3.23), we have the $L^{\infty}\left(L^{\infty}\right)$ error estimate

$$
\left\|u\left(t_{n}\right)-u_{h}^{n}\right\|_{L^{\infty}} \leq C|\ln h|^{\frac{1}{2}}\left(\left(\tilde{\rho}_{4}(\epsilon)\right)^{\frac{1}{2}}|\ln h|^{\frac{1}{2}} h+\left(\tilde{\rho}_{5}(\epsilon)\right)^{\frac{1}{2}} k\right) \quad \forall 1 \leq n \leq \ell .
$$

REMARK 3. The mesh constraints in Theorem 3.15 in [27] and (3.23) can be achieved by $h=C \epsilon^{p_{1}}$ and $k=C \epsilon^{p_{2}}$ for certain positive $p_{1}, p_{2}$. Hence, the $|\ln h| k^{2}$ decreases asymptoticly as $k^{2}$ when $\epsilon$ goes to zero.

5. Convergence of the Numerical Interface. In this section, we prove that the numerical interface defined as the zero level set of the Morley element interpolation of the solution $U^{n}$ converges to the moving interface of the Hele-Shaw problem under the assumption that the Hele-Shaw problem has a unique global (in time) classical solution. We first cite the following convergence result established in [2].

THEOREM 5.1. Let $\Omega$ be a given smooth domain and $\Gamma_{00}$ be a smooth closed hypersurface in $\Omega$. Suppose that the Hele-Shaw problem starting from $\Gamma_{00}$ has a unique smooth solution $\left(w, \Gamma:=\bigcup_{0 \leq t \leq T}\left(\Gamma_{t} \times\{t\}\right)\right)$ in the time interval $[0, T]$ such that $\Gamma_{t} \subseteq \Omega$ for all $t \in[0, T]$. Then there exists a family of smooth functions $\left\{u_{0}^{\epsilon}\right\}_{0<\epsilon \leq 1}$ which are uniformly bounded in $\epsilon \in(0,1]$ and $(x, t) \in \bar{\Omega}_{T}$, such that if $u^{\epsilon}$ solves the Cahn-Hilliard problem (1.1)-(1.3), then

(i) $\lim _{\epsilon \rightarrow 0} u^{\epsilon}(x, t)=\left\{\begin{array}{ll}1 & \text { if }(x, t) \in \mathcal{O} \\ -1 & \text { if }(x, t) \in \mathcal{I}\end{array}\right.$ uniformly on compact subsets, where $\mathcal{I}$ and $\mathcal{O}$ stand for the "inside" and "outside" of $\Gamma$; 
(ii) $\lim _{\epsilon \rightarrow 0}\left(\epsilon^{-1} f\left(u^{\epsilon}\right)-\epsilon \Delta u^{\epsilon}\right)(x, t)=-w(x, t)$ uniformly on $\bar{\Omega}_{T}$.

We are now ready to state the first main theorem of this section.

Theorem 5.2. Let $\left\{\Gamma_{t}\right\}_{t \geq 0}$ denote the zero level set of the Hele-Shaw problem and $U_{\epsilon, h, k}(x, t)$ denotes the piecewise linear interpolation in time of the numerical solution $u_{h}^{n}$, namely,

$$
U_{\epsilon, h, k}(x, t):=\frac{t-t_{n-1}}{k} u_{h}^{n}(x)+\frac{t_{n}-t}{k} u_{h}^{n-1}(x),
$$

for $t_{n-1} \leq t \leq t_{n}$ and $1 \leq n \leq M$. Then, under the mesh and starting value constraints of Theorem 4.6 and $k=O\left(h^{q}\right)$ with $0<q<1$, we have

(i) $U_{\epsilon, h, k}(x, t) \stackrel{\epsilon \searrow 0}{\longrightarrow} 1$ uniformly on compact subset of $\mathcal{O}$,

(ii) $U_{\epsilon, h, k}(x, t) \stackrel{\epsilon \searrow 0}{\longrightarrow}-1$ uniformly on compact subset of $\mathcal{I}$.

Proof. For any compact set $A \subset \mathcal{O}$ and for any $(x, t) \in A$, we have

$$
\begin{aligned}
\left|U_{\epsilon, h, k}-1\right| & \leq\left|U_{\epsilon, h, k}-u^{\epsilon}(x, t)\right|+\left|u^{\epsilon}(x, t)-1\right| \\
& \leq\left|U_{\epsilon, h, k}-u^{\epsilon}(x, t)\right|_{L^{\infty}\left(\Omega_{T}\right)}+\left|u^{\epsilon}(x, t)-1\right| .
\end{aligned}
$$

Theorem 4.7 infers that

$$
\left|U_{\epsilon, h, k}-u^{\epsilon}(x, t)\right|_{L^{\infty}\left(\Omega_{T}\right)} \leq C\left(\tilde{\rho}_{6}(\epsilon)\right)^{\frac{1}{2}} h^{q}|\ln h| .
$$

where $\tilde{\rho}_{6}(\epsilon)=\max \left\{\tilde{\rho}_{4}(\epsilon), \tilde{\rho}_{5}(\epsilon)\right\}$.

The first term on the right-hand side of (5.2) tends to 0 when $\epsilon \searrow 0$ (note that $h, k \searrow 0$, too). The second term converges uniformly to 0 on the compact set $A$, which is ensured by (i) of Theorem 5.1. Hence, the assertion (i) holds.

To show (ii), we only need to replace $\mathcal{O}$ by $\mathcal{I}$ and 1 by -1 in the above proof.

The second main theorem addresses the convergence of numerical interfaces.

Theorem 5.3. Let $\Gamma_{t}^{\epsilon, h, k}:=\left\{x \in \Omega ; U_{\epsilon, h, k}(x, t)=0\right\}$ be the zero level set of $U_{\epsilon, h, k}(x, t)$, then under the assumptions of Theorem 5.2, we have

$$
\sup _{x \in \Gamma_{t}^{\epsilon, h, k}} \operatorname{dist}\left(x, \Gamma_{t}\right) \stackrel{\epsilon \searrow 0}{\longrightarrow} 0 \quad \text { uniformly on }[0, T] \text {. }
$$

Proof. For any $\eta \in(0,1)$, define the tabular neighborhood $\mathcal{N}_{\eta}$ of width $2 \eta$ of $\Gamma_{t}$

$$
\mathcal{N}_{\eta}:=\left\{(x, t) \in \Omega_{T} ; \operatorname{dist}\left(x, \Gamma_{t}\right)<\eta\right\} .
$$

Let $A$ and $B$ denote the complements of the neighborhood $\mathcal{N}_{\eta}$ in $\mathcal{O}$ and $\mathcal{I}$, respectively,

$$
A=\mathcal{O} \backslash \mathcal{N}_{\eta} \quad \text { and } \quad B=\mathcal{I} \backslash \mathcal{N}_{\eta} .
$$

Note that $A$ is a compact subset outside $\Gamma_{t}$ and $B$ is a compact subset inside $\Gamma_{t}$. By Theorem 5.2, there exists $\epsilon_{1}>0$, which only depends on $\eta$, such that for any $\epsilon \in\left(0, \epsilon_{1}\right)$

$$
\begin{aligned}
& \left|U_{\epsilon, h, k}(x, t)-1\right| \leq \eta \quad \forall(x, t) \in A, \\
& \left|U_{\epsilon, h, k}(x, t)+1\right| \leq \eta \quad \forall(x, t) \in B .
\end{aligned}
$$


Now for any $t \in[0, T]$ and $x \in \Gamma_{t}^{\epsilon, h, k}$, from $U_{\epsilon, h, k}(x, t)=0$ we have

$$
\begin{aligned}
\left|U_{\epsilon, h, k}(x, t)-1\right| & =1 & \forall(x, t) & \in A, \\
\left|U_{\epsilon, h, k}(x, t)+1\right| & =1 & \forall(x, t) & \in B .
\end{aligned}
$$

(5.5) and (5.7) imply that $(x, t)$ is not in $A$, and (5.6) and (5.8) imply that $(x, t)$ is not in $B$, then $(x, t)$ must lie in the tubular neighborhood $\mathcal{N}_{\eta}$. Therefore, for any $\epsilon \in\left(0, \epsilon_{1}\right)$,

$$
\sup _{x \in \Gamma_{t}^{\epsilon, h, k}} \operatorname{dist}\left(x, \Gamma_{t}\right) \leq \eta \quad \text { uniformly on }[0, T] .
$$

The proof is complete.

6. Numerical experiments. In this section, we present two two-dimensional numerical tests to gauge the performance of the proposed fully discrete Morley finite element method for Cahn-Hilliard equation. The square domain $\Omega=[-1,1]^{2}$ is used in both tests.

Test 1. Consider the Cahn-Hilliard problem with an ellipse initial interface determined by $\Gamma_{0}: \frac{x^{2}}{0.36}+\frac{y^{2}}{0.04}=0$. The initial condition is chosen to have the form $u_{0}(x, y)=\tanh \left(\frac{d_{0}(x, y)}{\sqrt{2 \epsilon}}\right)$, where $d_{0}(x, y)$ denotes the signed distance from $(x, y)$ to the initial ellipse interface $\Gamma_{0}$ and $\tanh (t)=\left(e^{t}-e^{-t}\right) /\left(e^{t}+e^{-t}\right)$.

Figure 1 displays four snapshots at four fixed time points of the numerical interface with four different $\epsilon$ 's. Here time step size $k=1 \times 10^{-4}$ and space size $h=0.01$ are used. They clearly indicate that at each time point the numerical interface converges to the sharp interface $\Gamma_{t}$ of the Hele-Shaw flow as $\epsilon$ tends to zero. Note that this initial condition may not satisfy the General Assumption (GA) due to the singularity of the signed distance function. We will adopt a smooth initial condition in the later test.

Test 2. Consider the following initial condition, which is also adopted in [23],

$$
u_{0}(x, y)=\tanh \left(\left((x-0.3)^{2}+y^{2}-0.25^{2}\right) / \epsilon\right) \tanh \left(\left((x+0.3)^{2}+y^{2}-0.3^{2}\right) / \epsilon\right) .
$$

Table 1 and 2 show the errors of spatial $L^{2}, H^{1}$ and $H^{2}$ semi-norms and the rates of convergence at $T=0.0002$ and $T=0.001$. $\epsilon=0.08$ is used to generate the table. $k=1 \times 10^{-5}$ is chosen so that the error in time is relatively small to the error in space. The $L^{\infty}\left(H^{2}\right)$ norm error is in agreement with the convergence theorem, but $L^{\infty}\left(L^{2}\right)$ and $L^{\infty}\left(H^{1}\right)$ norm errors are one order higher than our theoretical results. We note that in [14], the second order convergence for both $L^{\infty}\left(L^{2}\right)$ and $L^{\infty}\left(H^{1}\right)$ norms are proved, whereas only $\frac{1}{\epsilon}$-exponential dependence can be derived.

\begin{tabular}{|l||c|c||c|c||c|c|}
\hline & $L^{\infty}\left(L^{2}\right)$ error & order & $L^{\infty}\left(H^{1}\right)$ error & order & $L^{\infty}\left(H^{2}\right)$ error & order \\
\hline$h=0.2 \sqrt{2}$ & 0.079659 & - & 1.761563 & - & 34.097686 & - \\
\hline$h=0.1 \sqrt{2}$ & 0.023142 & 1.7833 & 0.642870 & 1.4543 & 21.604986 & 0.6583 \\
\hline$h=0.05 \sqrt{2}$ & 0.007598 & 1.6067 & 0.183600 & 1.8080 & 11.783724 & 0.8746 \\
\hline$h=0.025 \sqrt{2}$ & 0.002151 & 1.8201 & 0.048042 & 1.9342 & 6.045416 & 0.9629 \\
\hline$h=0.0125 \sqrt{2}$ & 0.000557 & 1.9501 & 0.012167 & 1.9813 & 3.042138 & 0.9908 \\
\hline
\end{tabular}

TABLE 1

Spatial errors and convergence rates of Test 2: $\epsilon=0.08, k=1 \times 10^{-5}, T=0.0002$.

Figure 2 displays six snapshots at six fixed time points of the numerical interface with four different $\epsilon$. Again, they clearly indicate that at each time point the numerical interface converges to the sharp interface $\Gamma_{t}$ of the Hele-haw flow as $\epsilon$ tends to zero. 

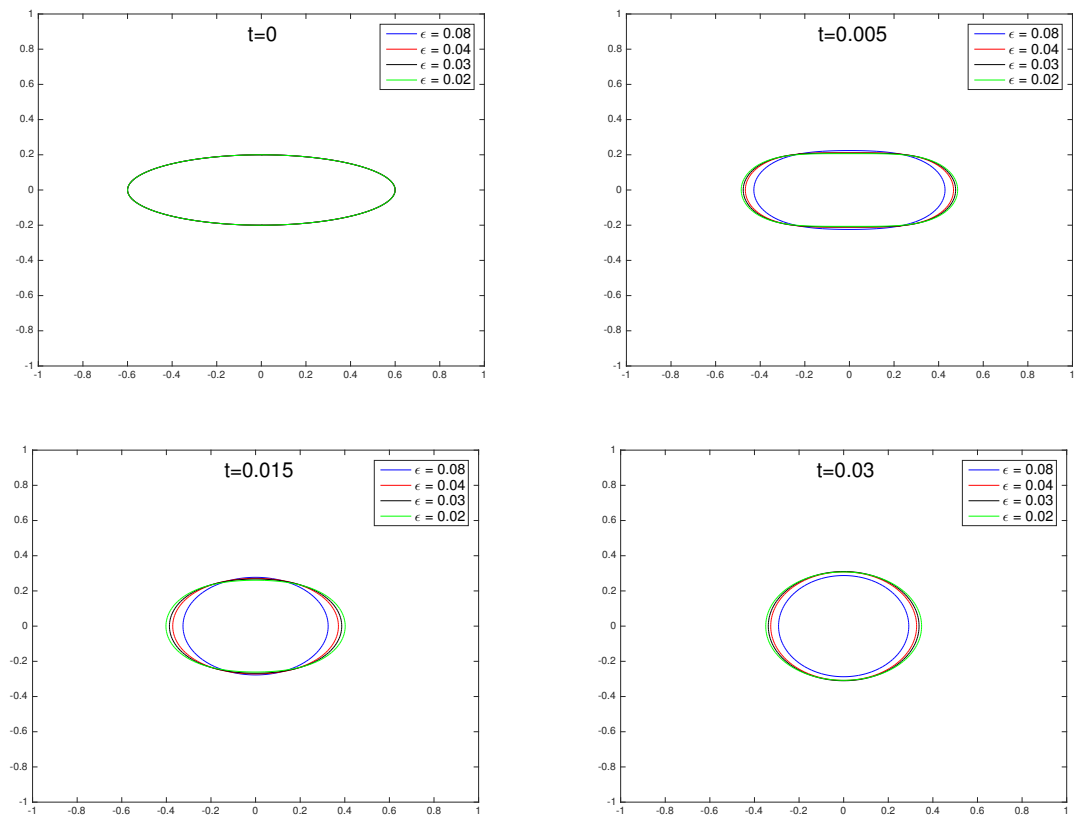

Fig. 1. Test 1: Snapshots of the zero-level sets of $u^{\epsilon, k}$ at $t=0,0.005,0.015,0.03$ and $\epsilon=$ $0.08,0.04,0.03,0.02$.

\begin{tabular}{|l||c|c||c|c||c|c|}
\hline & $L^{\infty}\left(L^{2}\right)$ error & order & $L^{\infty}\left(H^{1}\right)$ error & order & $L^{\infty}\left(H^{2}\right)$ error & order \\
\hline$h=0.2 \sqrt{2}$ & 0.137170 & - & 2.469582 & - & 43.008910 & - \\
\hline$h=0.1 \sqrt{2}$ & 0.032310 & 2.0859 & 0.710340 & 1.7977 & 23.320078 & 0.8831 \\
\hline$h=0.05 \sqrt{2}$ & 0.008830 & 1.8715 & 0.183932 & 1.9493 & 11.774451 & 0.9859 \\
\hline$h=0.025 \sqrt{2}$ & 0.002349 & 1.9103 & 0.046810 & 1.9743 & 5.927408 & 0.9902 \\
\hline$h=0.0125 \sqrt{2}$ & 0.000597 & 1.9746 & 0.011764 & 1.9924 & 2.970322 & 0.9968 \\
\hline
\end{tabular}

TABLE 2

Spatial errors and convergence rates of Test 2: $\epsilon=0.08, k=1 \times 10^{-5}, T=0.001$.

Acknowledgements. The authors Shuonan Wu and Yukun Li highly thank Professor Xiaobing Feng in the University of Tennessee at Knoxville for his motivation for this paper.

\section{REFERENCES}

[1] R. A. Adams and J. J. Fournier, Sobolev spaces, vol. 140, Elsevier, 2003.

[2] N. D. Alikakos, P. W. Bates, And X. Chen, Convergence of the Cahn-Hilliard equation to the Hele-Shaw model, Archive for Rational Mechanics and Analysis, 128 (1994), pp. 165-205.

[3] S. M. Allen and J. W. CAhn, A microscopic theory for antiphase boundary motion and its application to antiphase domain coarsening, Acta Metallurgica, 27 (1979), pp. 1085-1095.

[4] A. C. Aristotelous, O. Karakashian, and S. M. Wise, A mixed discontinuous Galerkin, convex splitting scheme for a modified Cahn-Hilliard equation and an efficient nonlinear multigrid solver, Discrete \& Continuous Dynamical Systems-Series B, 18 (2013).

[5] D. N. Arnold, F. Brezzi, B. Cockburn, and L. D. Marini, Unified analysis of discontinuous Galerkin methods for elliptic problems, SIAM Journal on Numerical Analysis, 39 (2002), pp. 1749-1779.

[6] S. Bartels, R. MÜller, And C. ORTner, Robust a priori and a posteriori error analysis for 

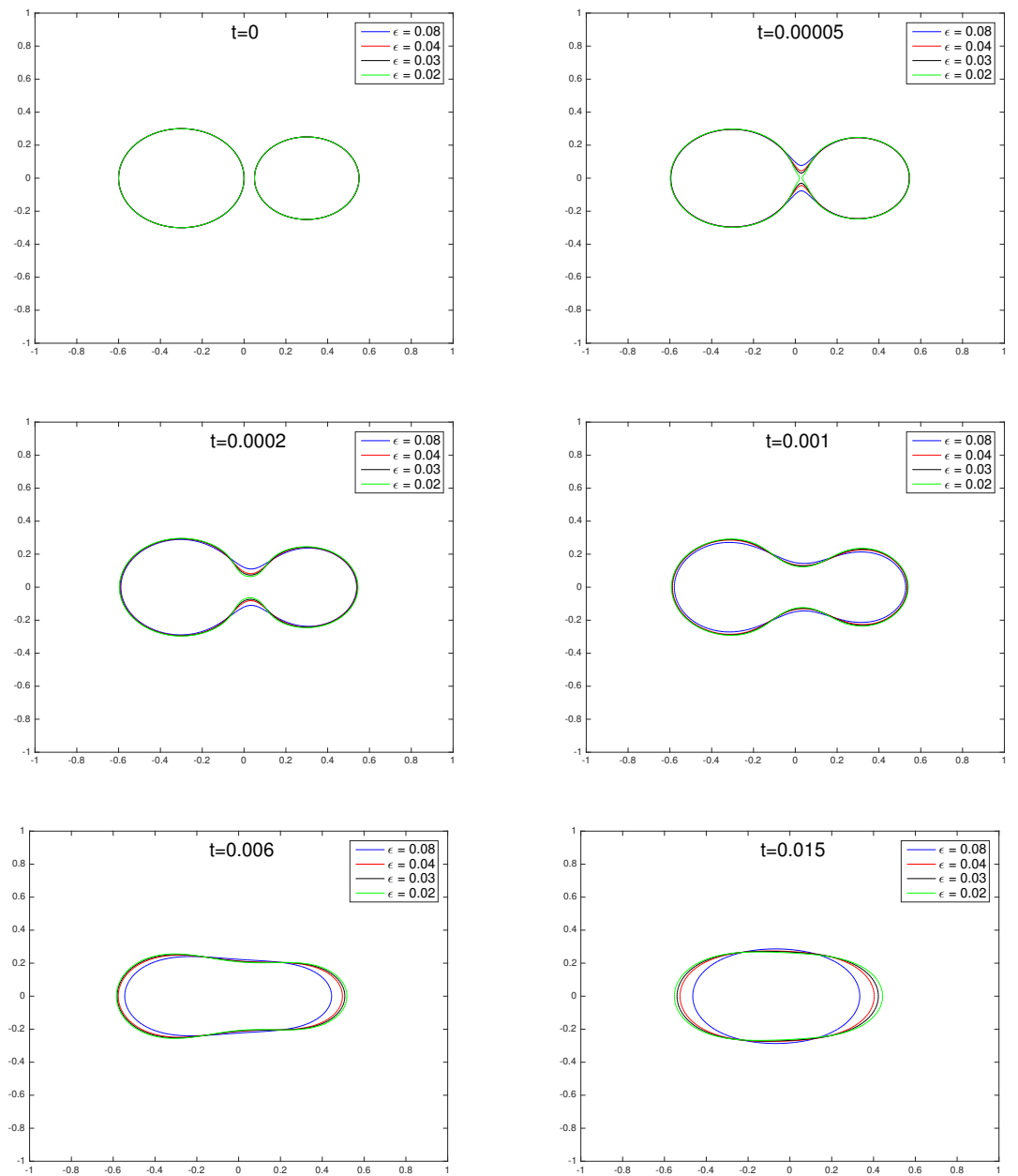

FIG. 2. Test 2: Snapshots of the zero-level sets of $u^{\epsilon, k}$ at $t=$ $0,0.00005,0.0002,0.001,0.006,0.015$ and $\epsilon=0.08,0.04,0.03,0.02$.

the approximation of Allen-Cahn and Ginzburg-Landau equations past topological changes, SIAM Journal on Numerical Analysis, 49 (2011), pp. 110-134.

[7] S. Brenner, Two-level additive Schwarz preconditioners for nonconforming finite element methods, Mathematics of Computation, 65 (1996), pp. 897-921.

[8] S. Brenner, Convergence of nonconforming multigrid methods without full elliptic regularity, Mathematics of Computation, 68 (1999), pp. 25-53.

[9] S. C. Brenner, Forty years of the Crouzeix-Raviart element, Numerical Methods for Partial Differential Equations, 31 (2015), pp. 367-396.

[10] S. C. Brenner, L.-Y. Sung, H. Zhang, and Y. Zhang, A Morley finite element method for the displacement obstacle problem of clamped Kirchhoff plates, Journal of Computational and Applied Mathematics, 254 (2013), pp. 31-42.

[11] J. W. Cahn And J. E. Hilliard, Free energy of a nonuniform system I, Interfacial free energy, Journal of Chemical Physics, 28 (1958), pp. 258-267.

[12] X. Chen, Spectrum for the Allen-Chan, Chan-Hillard, and phase-field equations for generic interfaces, Communications in Partial Differential Equations, 19 (1994), pp. 1371-1395.

[13] Q. Du AND R. A. Nicolaides, Numerical analysis of a continuum model of phase transition, SIAM Journal on Numerical Analysis, 28 (1991), pp. 1310-1322. 
[14] C. M. Elliott and D. A. French, A nonconforming finite-element method for the twodimensional Cahn-Hilliard equation, SIAM Journal on Numerical Analysis, 26 (1989), pp. 884-903.

[15] L. C. Evans, H. M. Soner, And P. E. Souganidis, Phase transitions and generalized motion by mean curvature, Communications on Pure and Applied Mathematics, 45 (1992), pp. 10971123.

[16] X. Feng And Y. LI, Analysis of symmetric interior penalty discontinuous Galerkin methods for the Allen-Cahn equation and the mean curvature flow, IMA Journal of Numerical Analysis, 35 (2014), pp. 1622-1651.

[17] X. Feng, Y. LI, And A. Prohl, Finite element approximations of the stochastic mean curvature flow of planar curves of graphs, Stochastic Partial Differential Equations: Analysis and Computations, 2 (2014), pp. 54-83.

[18] X. Feng, Y. LI, And Y. XING, Analysis of mixed interior penalty discontinuous Galerkin methods for the Cahn-Hilliard equation and the Hele-Shaw flow, SIAM Journal on Numerical Analysis, 54 (2016), pp. 825-847.

[19] X. Feng, Y. Li, AND Y. Zhang, Finite element methods for the stochastic Allen-Cahn equation with gradient-type multiplicative noise, SIAM Journal on Numerical Analysis, 55 (2017), pp. 194-216.

[20] X. Feng And A. Prohl, Numerical analysis of the Allen-Cahn equation and approximation for mean curvature flows, Numerische Mathematik, 94 (2003), pp. 33-65.

[21] X. Feng And A. Prohl, Error analysis of a mixed finite element method for the Cahn-Hilliard equation, Numerische Mathematik, 99 (2004), pp. 47-84.

[22] X. Feng and A. Prohl, Numerical analysis of the Cahn-Hilliard equation and approximation for the Hele-Shaw problem, Interfaces and Free Boundaries, 7 (2005), pp. 1-28.

[23] X. FEng AND H.-J. WU, A posteriori error estimates and an adaptive finite element approximations of the Cahn-Hilliard equation and the Hele-Shaw flow, Journal of Computational Mathematics, 26 (2008), pp. 767-796.

[24] T. Ilmanen Et AL., Convergence of the Allen-Cahn equation to Brakke's motion by mean curvature, Journal Differential Geometry, 38 (1993), pp. 417-461.

[25] M. Kovács, S. Larsson, and A. Mesforush, Finite element approximation of the CahnHilliard-Cook equation, SIAM Journal on Numerical Analysis, 49 (2011), pp. 2407-2429.

[26] Y. LI, Numerical methods for deterministic and stochastic phase field models of phase transition and related geometric flows, $\mathrm{PhD}$ thesis, The University of Tennessee, 2015.

[27] Y. LI, Error analysis of a fully discrete Morley finite element approximation for the CahnHilliard equation, arXiv preprint arXiv:1712.01338, (2017).

[28] B. E. Sтотн, Convergence of the Cahn-Hilliard equation to the Mullins-Sekerka problem in spherical symmetry, Journal of Differential Equations, 125 (1996), pp. 154-183.

[29] S. Wu AND J. Xu, Multiphase Allen-Cahn and Cahn-Hilliard models and their discretizations with the effect of pairwise surface tensions, Journal of Computational Physics, 343 (2017), pp. $10-32$.

[30] J. Xu, Y. Li, S. Wu, AND A. Bousquet, On the stability and accuracy of partially and fully implicit schemes for phase field modeling, arXiv preprint arXiv:1604.05402, (2016). 